Discussion Paper No. $\quad 580$

\title{
INFORMATION FEEDBACK IN
}

\section{A DYNAMIC TOURNAMENT}

Masaki Aoyagi

May 2003

The Institute of Social and Economic Research

Osaka University

6-1 Mihogaoka, Ibaraki, Osaka 567-0047, Japan 


\title{
Information Feedback in a Dynamic Tournament
}

\author{
MASAKI AOYAGI $\ddagger$
}

May 9, 2003

\begin{abstract}
This paper studies the problem of information revelation in a multi-stage tournament where the agents' effort in each stage gives rise to a stochastic performance signal privately observed by the principal. The principal controls the agents' effort incentive through the use of a feedback policy, which transforms his private information into a public announcement. The optimal feedback policy is one that maximizes the agents' expected effort. The paper identifies when the principal should use the no-feedback policy that reveals no information, or the full-feedback policy that reveals all his information.

Key words: tournament, mechanism, information revelation, Jensen's inequality.

Journal of Economic Literature Classification Numbers: C72, D82.
\end{abstract}

$\ddagger$ ISER, Osaka University. 


\section{Introduction}

As a prominent form of relative performance evaluation, tournaments have attracted considerable attention in economic theory. The main focus of the theory is on the size and allocation of rewards that would maximize the performance of the competing agents, and on the comparison of the relative incentive schemes against more general forms of contracts. Beginning with the seminal work of Lazear and Rosen (1981), a partial list of the literature on this subject includes Green and Stokey (1983), Nalebuff and Stiglitz (1983), Glazer and Hassin (1988), Gradstein and Konrad (1999), Moldovanu and Sela (2001), and others. In most models, a tournament is described as a static mechanism in which the agents' one-time effort decision determines their performance and hence the winner. In reality, however, many tournaments are more appropriately described as dynamic games: Agents make sequential effort decisions in multiple stages and the winner is determined by their overall performance. When a tournament is designed as a dynamic mechanism, one important issue arises concerning the control of information during the course of play. In other words, the design of a dynamic tournament should include strategic considerations on how information is revealed to the competing agents. In general, the timing and content of such information feedback should have a significant impact on the agents' effort incentive. Consider, for example, a tournament for job promotion within a firm. First, such a tournament is dynamic in nature and spans multiple stages. Second, workers' performance, which is typically measured by the combination of such factors as leadership, originality, ability to work in teams, etc., is often subjective and considered private information of his boss or the firm's personnel division. Research on performance management well recognizes that the effective inducement of the work incentive requires careful designing of a scheme through which such performance information is fed back to the worker. ${ }^{1}$

In this paper, we formulate a model of a dynamic tournament in which the principal receives private information about agents' performance, and then reveals as a feedback some or all of his information to the agents. The analysis is dual to that in the standard contest literature in that we fix prizes and focus exclusively on the effects of information. While strategic transmission of private information is a much studied subject in economic theory, no general understanding exists about how a mechanism should incorporate the use of the designer's private information. Existing theories provide varying intuitions as follows.

\footnotetext{
${ }^{1}$ See, for example, Williams (1998).
} 
In auction theory, the so-called linkage principle by Milgrom and Roberts (1982) asserts that under the affiliated distribution of signals, the seller's expected revenue is the highest when he is committed to revealing all of his private information to the bidders. ${ }^{2}$ In a related framework, Milgrom (1981) shows that the seller of a good maximizes his payoff by revealing all his private information to the buyer if it is affiliated with the quality of his good. In some other situations, however, it is shown that the intuition furnished by the linkage principle fails to hold: Kaplan and Zamir (2000) analyze the problem of an auctioneer privately informed about bidders' valuations. In an independent private values framework, they find that the auctioneer is better off revealing the maximum of the valuations than fully revealing his information. In a model of twice-repeated common-value auctions with affiliated signals, de-Frutos and Rosenthal (1998) show that the auctioneer's expected revenue (over two auctions) is lower when information about stage 1 bids is made public than when it is not. ${ }^{3}$

The literature on dynamic models of a race also provides a closely related observation in the discussion of the closed- and open-loop formats. ${ }^{4}$ The open-loop format reveals no information to the players during a competition, whereas the closed-loop format reveals one another's move publicly and instantaneously. It is often argued that the players tend to slack off in the closed-loop format since, when one player has a small lead over the others, the followers cannot catch up (in expected terms) with the leader by making the same level of effort as him. In some circumstances, players stop making effort as soon as a small lead is established. ${ }^{5}$

It is important to understand that information feedback has two separate effects on the agents' incentives. First, the revealed information influences the agents' incentives by changing their beliefs. This is true irrespective of whether the principal's private information is given exogenously as in the case of the linkage principle, or is generated endogenously by the agents' own actions as in our model. We call this the ex post effect of information feedback. On the other hand, when the private information is generated endogenously, each agent will choose their actions strategically so as to influence the content

${ }^{2}$ A probability distribution is affiliated if the joint density function is log-supermodular. ${ }^{3}$ Perry and Reny (1999) report the failure of the linkage principle in a multi-object auction based on an entirely different logic.

${ }^{4}$ See, for example, Harris and Vickers (1985), and Fudenberg et al. (1983). Radner (1985) also makes a related observation in the context of a repeated principal-agent game.

${ }^{5}$ Such a phenomenon is referred to as $\epsilon$-preemption in Fudenberg et al. (1983). 
of the revealed information. For example, agents may exert extra effort in early stages to take the leading position and discourage opponents. We call this the strategic effect of information feedback. One interpretation of the finding of de-Frutos and Rosenthal (1998) is that these two effects may offset each other.

In our model of a multi-stage tournament, agents' performance in each stage is stochastically related to their effort in that stage. The principal privately observes their performance realization after each stage, and reveals some or all of his private information to the agents before the next stage. The principal's feedback policy transforms the raw observation of the agents' performance into a public announcement. In our terminology, the closed-loop and open-loop formats described above correspond to the full-feedback and no-feedback policies, respectively. ${ }^{6}$ The principal is free to choose any feedback policy and publicly announces its use before the tournament. For example, he may use a hybrid policy that reveals full information for some signal realizations but no information for others. ${ }^{7}$ We assume that the principal is committed to his feedback policy for any realization of the private signal. The optimal feedback policy is one that maximizes the principal's payoff which is an increasing function of the agents' efforts. As discussed below, we find that whether he should reveal more information or not depends critically on the functional form of the agents' disutility of effort.

A more detailed description of our model is as follows: Two agents compete in a tournament over $T$ stages. The agent with the higher performance at the end of stage $T$ wins and is awarded a prize of a fixed value such as a promotion to a higher job rank. In each stage $t$, two agents each choose an effort level $a_{t}$, which is observed by neither the principal nor the opponent. The agents' cost function of effort is time-separable and can be expressed as the sum of stage-cost functions, which are assumed to be all strictly convex. The score in stage $t$ is the difference between the performance levels of the two agents and equals the sum of the difference between their effort levels and a random noise term. The principal privately observes the score, and makes a public announcement about it at the end of stage $t$. Conditional on the announcement, the agents update their inference about the score and decide on their effort levels in subsequent stages. We study how the choice of a feedback policy affects the agents' effort levels in a pure perfect Bayesian equilibrium (PBE) of this dynamic game.

${ }^{6}$ Alternatively, the no-feedback policy can be interpreted as the simultaneous implementation of multiple one-shot tournaments.

${ }^{7}$ Under such a policy, of course, "no announcement" also has an informational content. 
The main differences between the present model and the models of auctions and dynamic races mentioned above are as follows. First, effort is a continuous variable chosen from the set of real numbers. This in particular implies that an agent can make high effort in any single stage and leapfrog his opponent. This possibility is often precluded in models of a race. Second, agents are symmetric in ability and performance is a noisy outcome of effort. This is in contrast to the incomplete information approach in the auction literature where the agents' performance level (bid) is a deterministic function of their private type (value or signal). ${ }^{8}$

The paper presents sufficient conditions for the existence of a perfect Bayesian equilibrium and derives effort levels on the equilibrium path. It then proceeds to characterize the optimal feedback policy. In short, revealing more information is better for the principal when the marginal cost of effort is concave, and the converse is true when the marginal cost is convex. More specifically, the following observations are made for the basic model with two stages $(T=2)$ : When the stage 2 marginal cost function of effort is convex, the no-feedback policy is optimal in the class of feedback policies that admit a symmetric PBE. On the other hand, the full-feedback policy is optimal in the same class when the marginal cost function is concave. Under a stronger condition on the distribution of the noise, the optimality of the no-feedback and full-feedback policies in the respective cases is extended for a possibly asymmetric PBE. The similar conclusion holds for a symmetric PBE of a general $T$-stage tournament. When the marginal cost of effort is convex in each stage, the no-feedback policy is optimal. When it is concave, on the other hand, the optimal policy is one that reveals the most information within the class of feedback policies that admit a symmetric PBE. ${ }^{9}$

The intuition for the above results for the two-stage model is as follows: As is standard, the agents' effort choice in each stage is such that its marginal disutility is balanced by the marginal increment in the probability of winning. It can be readily verified that the marginal increment in the probability of winning from stage 2 effort equals the conditional expectation of a function of the stage 1 score given the public announcement. Note in particular that the principal's feedback policy determines the coarseness of the condi-

\footnotetext{
${ }^{8}$ In this case, the main emphasis would be on strategic signaling of the private type through the revealed information.

${ }^{9}$ The full-feedback policy does not admit a symmetric equilibrium when $T \geq 3$. However, there exists a policy admitting a symmetric PBE that reveals effectively as much information as the full-feedback policy.
} 
tioning filtration. As for the stage 2 effort, its expected value equals the (unconditional) expectation of the above conditional expectation inverted by the stage 2 marginal cost function. When (the inverse of) the stage 2 marginal cost function is convex or concave, therefore, Jensen's inequality yields an ordering over various feedback policies according to the expected effort they induce. As for the stage 1 effort, on the other hand, it can be seen that its marginal disutility in equilibrium equals the expected marginal disutility from stage 2 effort. ${ }^{10}$ In other words, the marginal disutility of stage 1 effort is set equal to the unconditional expectation of the above function of the stage 1 score, which is independent of the feedback policy by the law of iterated expectation. Therefore, the stage 1 effort is constant under any feedback policy. Combination of these observations leads to the desired conclusion.

As seen from the above discussion, the optimal feedback policy often takes a simple but extreme form. What is important to note is the sensitivity of the optimal solution to the specification of the parameters of the model. For example, revealing no information is optimal in some cases, but is least desirable in others. Such sensitivity may in part explain the variations in the intuitions obtained from the existing models of information revelation as discussed above. On the other hand, it is also interesting to note that information feedback does not matter under the common assumption of quadratic cost functions, or equivalently, linear marginal cost functions. In this case, not only are the no-feedback and full-feedback policies optimal, but also any feedback policy induces the same expected effort.

In line with the standard assumption of the mechanism design literature, we assume that the principal is publicly committed to his feedback policy for any realization of the performance score. ${ }^{11}$ The conclusion would be very different without such commitment as a feedback policy must then be chosen subject to the sequential rationality requirement. ${ }^{12}$ We also assume that the principal's announcement is public. In some applications, it may be more appropriate to suppose that he can send a private message to each agent. Mares and Harstad (2002) show in their common-value auction model that an auctioneer may

${ }^{10}$ Although intuitive, this cannot be assumed a priori because of the strategic effect of stage 1 effort mentioned above. For example, agents may choose to exert larger effort in stage 1 in order to preempt the leading position.

${ }^{11}$ For example, an auctioneer does not sell his good below the reserve price even if no bid exceeds it.

${ }^{12}$ For example, Kaplan and Zamir (2000) find that the auctioneer without a commitment power cannot exploit his private information. 
be better off revealing his private information in a non-public way. Such might as well be the case in the present model. However, the analysis of private feedback of information is difficult as it induces a fundamental asymmetry in the agents' effort choice. This is left as a future exercise.

The paper is organized as follows: In the next section, we develop the basic framework for the two-stage model. Section 3 characterizes a PBE and provides sufficient conditions for its existence. In Section 4, the analysis of the optimal feedback policy is given. An extension to the general $T$-stage model is presented in Section 5 .

\section{Model of a Tournament}

Two risk neutral agents $i=1,2$ compete in two stages. In each stage, the agents' effort gives rise to a stochastic "score," which indicates their relative performance. At the end of stage 2, the principal aggregates the scores from both stages to determine the winner. ${ }^{13}$

Formally, suppose that agent $i$ 's effort $a_{t}^{i}$ in stage $t$ is chosen from the set $\mathbf{R}_{+}$of non-negative real numbers. The stage $t$ score $x_{t}$ is a random variable whose distribution depends on the effort levels $a_{t}^{1}$ and $a_{t}^{2}$ of both agents in stage $t$. More specifically, we assume that $x_{t}=a_{t}^{1}-a_{t}^{2}+\zeta_{t}$ for a real-valued random variable $\zeta_{t}$. In other words, the score $x_{t}$ represents agent 1 's lead over agent 2 , and is stochastically related to the difference between their effort levels. Let $\phi_{t}$ be the density of $\zeta_{t}$ over $\mathbf{R}$, and denote by $\Phi_{t}$ the corresponding cumulative distribution. We assume that $\phi_{t}$ is strictly positive and twice continuously differentiable, and symmetric around zero in the sense that $\phi_{t}(x)=\phi_{t}(-x)$ for any $x \in \mathbf{R}$. We also assume that $\zeta_{1}$ and $\zeta_{2}$ are independent. Note that the density of $x_{t}$ under the action profile $a_{t}=\left(a_{t}^{1}, a_{t}^{2}\right)$ is given by

$$
\phi_{t}\left(x_{t}-a_{t}^{1}+a_{t}^{2}\right) .
$$

The (aggregate) score $x$ is the sum of scores in stages 1 and 2: $x=x_{1}+x_{2}$. Agent 1 wins if $x>0$, and agent 2 wins if $x<0$. Each agent wins with equal probability in the (probability zero) event of a tie $x=0$.

Each agent derives one unit of positive utility from the winning prize (e.g., promotion to a higher job rank), and incurs disutility from effort. The cost of effort in stage $t$ is

${ }^{13}$ While this is a special case of the more general model discussed in Section 5, the two-stage setting allows for a clearer prensetation and more permissive conditions. 
described by a twice differentiable cost function $c_{t}: \mathbf{R}_{+} \rightarrow \mathbf{R}_{+}$. Accordingly, agent $i$ 's overall utility equals $1-\sum_{t=1}^{2} c_{t}\left(a_{t}^{i}\right)$ if he wins, and $-\sum_{t=1}^{2} c_{t}\left(a_{t}^{i}\right)$ otherwise. The principal's payoff, on the other hand, is a function of each agent's effort levels in the two stages: $V\left(a_{1}^{1}, a_{1}^{2}, a_{2}^{1}, a_{2}^{2}\right)$. The function $V: \mathbf{R}_{+}^{4} \rightarrow \mathbf{R}$ is assumed to be increasing $\left(V(\hat{a}) \geq V(a)\right.$ if $\hat{a}_{t}^{i} \geq a_{t}^{i}$ for each $\left.t, i=1,2\right)$ and symmetric with respect to the agents $\left(V(\hat{a})=V(a)\right.$ if $\hat{a}_{t}^{1}=a_{t}^{2}$ and $\hat{a}_{t}^{2}=a_{t}^{1}$ for $\left.t=1,2\right)$. Note that the principal's payoff may in general contain more information than his private signal $x$ about the agents' efforts. In line with our assumption that the winner is determined based only on $x$, we suppose that the principal observes his payoff only after the winner has been determined. Each agent's effort $a_{t}^{i}$ is his private information and observed by neither the principal nor the other agent. On the other hand, the principal privately observes the score $x_{t}$ in each stage $t$ and can credibly reveal either whole or part of his private information $x_{1}$ after stage 1. Specifically, suppose that the principal makes a public announcement $y$ about $x_{1}$ at the end of stage 1. Let $Y$ be the set of possible announcements. A feedback policy (or simply a policy) is a measurable mapping $f: \mathbf{R} \rightarrow Y$, which chooses the announcement $y=f\left(x_{1}\right)$ as a function of the score $x_{1}$. The announcement $y$ is credible in the sense that the principal publicly announces $f$ at the beginning and is committed to it for any realization of $x_{1}$. The principal chooses a feedback policy so as to maximize his expected payoff. For simplicity, our analysis will be restricted to deterministic feedback policies. The paper's conclusions do not change with the introduction of a stochastic feedback policy, which chooses the announcement $y$ as a function of $x_{1}$ and some (exogenous) random variable.

Little restriction is placed on the nature of the public announcement $y$. For example, each announcement $y \in Y$ may simply contain the name of the leader, or it may be an interval in $\mathbf{R}$ which indicates the range of $x_{1}$. Without loss of generality, we assume that $f$ is a surjection by choosing $Y=\left\{f\left(x_{1}\right): x_{1} \in \mathbf{R}\right\}$.

As mentioned in the Introduction, some simple feedback policies will play an import role in our analysis. In particular, the no-feedback policy sends the same message regardless of $x_{1}$, and the full-feedback policy reveals $x_{1}$ completely. Given any announcement $y \in Y$, let $f^{-1}(y)=\left\{x_{1} \in \mathbf{R}: f\left(x_{1}\right)=y\right\}$ denote the inverse image of $\{y\}$ under $f$. The feedback policy $f$ is regular if for any $y \in Y, f^{-1}(y) \subset \mathbf{R}$ either has positive (Lebesgue) measure, or is countable. Most "natural" policies, including the full-feedback and no-feedback policies, are regular. ${ }^{14}$ In what follows, we will restrict attention to regular feedback policies.

\footnotetext{
${ }^{14}$ Feedback policy $f$ fails to be regular if $f^{-1}(y)$ is, for example, the Cantor set for some
} 
Given any policy $f$, agent $i$ 's history $h^{i}$ after stage 1 is the information available to agent $i$ at the end of stage $1: h^{i}$ consists of his own effort choice $a_{1}^{i}$, and the public announcement $y$ by the principal. Agent $i$ 's (pure) strategy $\sigma^{i}$ is a pair $\left(\sigma_{1}^{i}, \sigma_{2}^{i}\right)$, where $\sigma_{1}^{i} \in \mathbf{R}_{+}$is the effort choice for stage 1 , and $\sigma_{2}^{i}: \mathbf{R}_{+} \times Y \rightarrow \mathbf{R}_{+}$is a mapping that specifies the stage 2 effort after each possible history $h^{i}=\left(a_{1}^{i}, y\right)$. Given the starategy profile $\sigma$, let $\pi_{2}^{i}\left(a_{2}^{i} \mid \sigma, h_{1}^{i}\right)$ denote agent $i$ 's expected payoff in stage 2 when he chooses $a_{2}^{i}$ in stage 2 , his history in stage 1 is $h_{1}^{i}$, and agent $j$ plays according to the strategy $\sigma^{j}$ in both stages. Likewise, let $\pi_{1}^{i}\left(a_{1}^{i} \mid \sigma\right)$ denote agent $i$ 's expected payoff in stages 1 and 2 when he chooses $a_{1}^{i}$ in stage 1 and plays according to $\sigma_{2}^{i}$ in stage 2 , and agent $j$ plays according to $\sigma^{j}$ in both stages. In view of the fact that the distribution $\phi_{1}$ has full support, we define a strategy profile $\sigma=\left(\sigma^{1}, \sigma^{2}\right)$ to be a (pure) perfect Bayesian equilibrium (PBE) if for $i=1,2$,

$$
\begin{aligned}
& \pi_{1}^{i}\left(\sigma_{1}^{i} \mid \sigma\right) \geq \pi_{1}^{i}\left(a_{1}^{i} \mid \sigma\right) \text { for any } a_{1}^{i} \in \mathbf{R}_{+}, \text {and } \\
& \pi_{2}^{i}\left(\sigma_{2}^{i}\left(h_{1}^{i}\right) \mid \sigma, h_{1}^{i}\right) \geq \pi_{2}^{i}\left(a_{2}^{i} \mid \sigma, h_{1}^{i}\right) \text { for any } a_{2}^{i} \in \mathbf{R}_{+} \text {and } h_{1}^{i} \in \mathbf{R}_{+} \times Y .
\end{aligned}
$$

\section{A Perfect Bayesian Equilibrium}

We assume that the marginal cost of effort in each stage is increasing:

Assumption 1: For $t=1$, 2, the cost function $c_{t}: \mathbf{R}_{+} \rightarrow \mathbf{R}_{+}$satisfies $c_{t}^{\prime}(0)=0$ and $\inf _{a \in \mathbf{R}_{+}} c_{t}^{\prime \prime}(a)>0$.

Let a regular feedback policy $f$ be given. We denote by $g_{1}^{\sigma}\left(x_{1} \mid y\right)$ the conditional density of $x_{1}$ given the stage 1 effort profile $\sigma_{1}=\left(\sigma_{1}^{1}, \sigma_{1}^{2}\right)$ and the public announcement $y$. For $x_{1} \in f^{-1}(y)$, it can be expressed as

$$
g_{1}^{\sigma}\left(x_{1} \mid y\right)=\frac{\phi_{1}\left(x_{1}-\sigma_{1}^{1}+\sigma_{1}^{2}\right)}{\int_{f^{-1}(y)} \phi_{1}\left(x_{1}^{\prime}-\sigma_{1}^{1}+\sigma_{1}^{2}\right) d x_{1}^{\prime}} \quad \text { or } \quad \frac{\phi_{1}\left(x_{1}-\sigma_{1}^{1}+\sigma_{1}^{2}\right)}{\sum_{x_{1}^{\prime} \in f^{-1}(y)} \phi_{1}\left(x_{1}^{\prime}-\sigma_{1}^{1}+\sigma_{1}^{2}\right)}
$$

depending on whether $f^{-1}(y)$ has positive measure or is countable. ${ }^{15}$ Recall that the convolution of $\phi_{1}$ and $\phi_{2}$, denoted $\phi_{1} * \phi_{2}$, is defined by

$$
\left(\phi_{1} * \phi_{2}\right)(x)=\int_{\mathbf{R}} \phi_{1}(x-u) \phi_{2}(u) d u .
$$

For any strategy profile $\sigma$, let $\sigma_{2,0}^{i}(y)=\sigma_{2}^{i}\left(\sigma_{1}^{i}, y\right)$ for $y \in Y$. In other words, $\sigma_{2,0}^{i}(y)$ is agent $i$ 's stage 2 effort along the path of play. The following theorem characterizes the effort level chosen in any pure PBE.

$y$.

${ }^{15}$ Note that $g_{1}^{\sigma}$ depends only on the stage 1 profile $\sigma_{1}$ and not on the stage 2 profile $\sigma_{2}$. 
Theorem 1. Suppose that Assumption 1 holds and that $\sup _{x \in \mathbf{R}}\left|\phi_{2}^{\prime}(x)\right|<\inf _{a \in \mathbf{R}_{+}} c_{2}^{\prime \prime}(a)$. If $\sigma$ is a pure PBE under any feedback policy $f$, then for any $y \in Y$,

$$
\sigma_{2,0}^{1}(y)=\sigma_{2,0}^{2}(y)=\alpha_{2}\left(\sigma_{1}, y\right) \equiv\left(c_{2}^{\prime}\right)^{-1}\left(E^{\sigma}\left[\phi_{2}\left(\tilde{x}_{1}\right) \mid y\right]\right) .
$$

If, in addition, $\sigma_{1}^{1}, \sigma_{1}^{2}>0$, then

$$
\left\{\begin{array}{l}
c_{1}^{\prime}\left(\sigma_{1}^{1}\right)=\left(\phi_{1} * \phi_{2}\right)\left(\sigma_{1}^{1}-\sigma_{1}^{2}\right)+\int_{\mathbf{R}} c_{2}\left(\alpha_{2}\left(\sigma_{1}, f\left(x_{1}\right)\right)\right) \phi_{1}^{\prime}\left(x_{1}-\sigma_{1}^{1}+\sigma_{1}^{2}\right) d x_{1}, \\
c_{1}^{\prime}\left(\sigma_{1}^{2}\right)=\left(\phi_{1} * \phi_{2}\right)\left(\sigma_{1}^{1}-\sigma_{1}^{2}\right)-\int_{\mathbf{R}} c_{2}\left(\alpha_{2}\left(\sigma_{1}, f\left(x_{1}\right)\right)\right) \phi_{1}^{\prime}\left(x_{1}-\sigma_{1}^{1}+\sigma_{1}^{2}\right) d x_{1} .
\end{array}\right.
$$

Proof: See the Appendix. //

It should be noted that in any PBE, the agents' stage 2 efforts are symmetric for any realization of the public announcement whether the stage 1 efforts are symmetric or not. It should be noted that the stage 2 effort is determined through the standard marginal consideration: It balances the increment in the probability of winning and the cost of one additional unit of effort. (1) has the following implication. Suppose for simplicity that $f$ is the full-feedback policy: $f\left(x_{1}\right)=x_{1}$. In this case, $\sigma_{2,0}^{i}\left(x_{1}\right)=\left(c_{2}^{\prime}\right)^{-1}\left(\phi_{2}\left(x_{1}\right)\right)$ as is readily verified. It follows that the agents make the highest stage 2 effort when $x_{1}$ is such that $\phi_{2}\left(x_{1}\right)$ is the largest. When $\phi_{2}$ is a unimodal distribution such as the normal distribution, this means that the stage 2 effort is maximal when the stage 1 score is $x_{1}=0$ and decreases with $\left|x_{1}\right|$. This supports the standard intuition that the closer the competition, the more effort the agents exert. Note, however, that this intuition no longer holds when, for example, $\phi_{2}$ is bimodel so that $\phi_{2}(x)=\phi_{2}(-x)>\phi_{2}(0)$ for some $x>0$.

The next theorem identifies a sufficient condition for the existence of a pure PBE.

Theorem 2. Suppose that Assumption 1 holds. There exists $\epsilon>0$ such that if

$$
\sup _{x \in \mathbf{R}} \phi_{2}(x), \sup _{x \in \mathbf{R}}\left|\phi_{2}^{\prime}(x)\right|, \int_{\mathbf{R}}\left|\phi_{1}^{\prime \prime}(x)\right| d x \text {, and } \int_{\mathbf{R}} \frac{\phi_{1}^{\prime}(x)^{2}}{\phi_{1}(x)} d x<\epsilon,
$$

then given any feedback policy $f$, there exists a pure PBE under $f$ if (2) has a solution $\sigma_{1}=\left(\sigma_{1}^{1}, \sigma_{1}^{2}\right) \geq 0$.

Proof: See the Appendix. //

The conditions involving $\phi_{t}$ in Theorems 1 and 2 all indicate that the performance score is a noisy signal of agents' effort. If, for example, $\zeta_{t}$ has the normal distribution 
$N\left(0, \sigma_{t}^{2}\right)(t=1,2)$, then these conditions hold whenever the variances $\sigma_{1}^{2}$ and $\sigma_{2}^{2}$ are sufficiently large: In fact, note that $\sup _{x \in \mathbf{R}} \phi_{2}(x)=\frac{1}{\sqrt{2 \pi} \sigma_{2}}, \sup _{x \in \mathbf{R}}\left|\phi_{2}^{\prime}(x)\right|=\frac{1}{\sqrt{2 \pi} \sigma_{2}^{2}} e^{-1 / 2}$,

$$
\int_{\mathbf{R}}\left|\phi_{1}^{\prime \prime}(x)\right| d x=\frac{1}{\sqrt{2 \pi} \sigma_{1}} \int_{\mathbf{R}}\left|-\frac{1}{\sigma_{1}^{2}} e^{-x^{2} / 2 \sigma_{1}^{2}}+\frac{x^{2}}{\sigma_{1}^{4}} e^{-x^{2} / 2 \sigma_{1}^{2}}\right| d x \leq \frac{2}{\sigma_{1}^{2}},
$$

and

$$
\int_{\mathbf{R}} \frac{\phi_{1}^{\prime}(x)^{2}}{\phi_{1}(x)} d x=\frac{1}{\sigma_{1}^{2}}
$$

It should be noted that high noise is a standard requirement for the existence of an equilibrium in a tournament model where performance is stochastically related to effort. ${ }^{16}$ Intuitively, if the noise is too small, then any infinitesimal increase in effort results in almost sure winning, making it impossible for the marginal equation to hold.

It should also be emphasized that in Theorem 2, the noise level required for the existence of an equilibrium is independent of a particular feedback policy $f$.

In what follows, we assume for simplicity that $Y$ is a vector space and normalize $f(0)=0 \in Y$. With this standardization, we say that a feedback policy $f$ is odd if $f(x)=-f(-x)$ for any $x \in \mathbf{R}$ and even if $f(x)=f(-x)$ for any $x \in \mathbf{R}$. Intuitively, if $f$ is odd, then the inference drawn from the announcement when agent $i$ leads agent $j$ in stage 1 is the exact opposite of that when their positions are reversed. On the other hand, if $f$ is even, then the announcement is the same regardless of the identity of the leader as long as the size of the lead is the same. For example, the full-feedback policy $f(x)=x$ is odd (but not even), whereas the no-feedback policy $f(x) \equiv 0$ is the only policy that is both odd and even.

We say that a strategy profile $\sigma$ is symmetric if the two agents always choose the same effort level on the path: $\sigma_{1}^{1}=\sigma_{1}^{2}$ and $\sigma_{2,0}^{1}(y)=\sigma_{2,0}^{2}(y)$ for any $y \in Y$. We now show that every even or odd policy admits a symmetric PBE. By summing the two equations of (2), we see that the only solution to (2) (if any) compatible with $\sigma_{1}^{1}=\sigma_{1}^{2}$ is

$$
\sigma_{1}^{1}=\sigma_{1}^{2}=a_{1}^{*} \equiv\left(c_{1}^{\prime}\right)^{-1}\left(\left(\phi_{1} * \phi_{2}\right)(0)\right)
$$

The following theorem shows that this indeed corresponds to an equilibrium when $f$ is either odd or even.

\footnotetext{
${ }^{16}$ See, for example, Nalebuff and Stiglitz (1983).
} 
Theorem 3. Suppose that Assumption 1 holds and that $\left(^{*}\right)$ holds for a sufficiently small $\epsilon>0$. If $f$ is either odd or even, then there exists a unique symmetric pure PBE $\sigma$. Furthermore, for $\alpha_{2}$ defined in (1) and $a_{1}^{*}$ defined in (3), $\sigma$ satisfies

$$
\sigma_{1}^{1}=\sigma_{1}^{2}=a_{1}^{*}
$$

and

$$
\sigma_{2,0}^{1}(y)=\sigma_{2,0}^{2}(y)=\alpha_{2}\left(\sigma_{1}, y\right) \quad \text { for any } y \in Y \text {. }
$$

Proof: See the Appendix. //

It should be noted that the stage 1 effort $a_{1}^{*}$ in the symmetric pure PBE is independent of the feedback policy $f$. Furthermore, the expected marginal cost in stage 2 equals the marginal cost in stage 1 since

$$
\begin{aligned}
E^{\sigma}\left[c_{2}^{\prime}\left(\alpha_{2}\left(\sigma_{1}, \tilde{y}\right)\right)\right] & =E^{\sigma}\left[E^{\sigma}\left[\phi_{2}\left(\tilde{x}_{1}\right) \mid \tilde{y}\right]\right] \\
& =E^{\sigma}\left[\phi_{2}\left(\tilde{x}_{1}\right)\right] \\
& =\left(\phi_{1} * \phi_{2}\right)(0) \\
& =c_{1}^{\prime}\left(a_{1}^{*}\right)
\end{aligned}
$$

by the law of iterated expectation. The following facts about the no-feedback and fullfeedback policies are immediate consequences of the above theorem.

Proposition 4. Suppose that Assumption 1 holds. If $\sigma$ is the (unique) symmetric pure $P B E$ under the no-feedback policy, then $\sigma_{1}^{1}=\sigma_{1}^{2}=a_{1}^{*}$ and

$$
\sigma_{2,0}^{1}(y)=\sigma_{2,0}^{2}(y)=a_{2}^{*} \equiv\left(c_{2}^{\prime}\right)^{-1}\left(\left(\phi_{1} * \phi_{2}\right)(0)\right) \quad \text { for any } y \in Y \text {. }
$$

Likewise, if $\sigma$ is the (unique) symmetric pure PBE under the full-feedback policy, then $\sigma_{1}^{1}=\sigma_{1}^{2}=a_{1}^{*}$ and

$$
E^{\sigma}\left[\sigma_{2,0}^{i}(\tilde{y})\right]=\int_{\mathbf{R}}\left(c_{2}^{\prime}\right)^{-1}\left(\phi_{2}\left(x_{1}\right)\right) \phi_{1}\left(x_{1}\right) d x_{1} .
$$

When $\left(c_{2}^{\prime}\right)^{-1}$ is concave or convex, Proposition 4 allows us to rank the no-feedback and full-feedback policies in terms of the expected stage 2 effort they induce in the symmetric PBE: When $\left(c_{2}^{\prime}\right)^{-1}$ is concave, Jensen's inequality implies that

$$
\int_{\mathbf{R}}\left(c_{2}^{\prime}\right)^{-1}\left(\phi_{2}\left(x_{1}\right)\right) \phi_{1}\left(x_{1}\right) d x_{1} \leq\left(c_{2}^{\prime}\right)^{-1}\left(\int_{\mathbf{R}} \phi_{2}\left(x_{1}\right) \phi_{1}\left(x_{1}\right) d x_{1}\right)=a_{2}^{*} .
$$


The reverse inequality holds when $\left(c_{2}^{\prime}\right)^{-1}$ is convex. The next section presents a generalization of these observations.

\section{Optimal Feedback Policy}

In this section, we will study the principal's expected payoff in the pure PBE as identified in Theorems 1-3. For this, we need to make the following assumption about the principal's payoff function:

Assumption 2: $V\left(a_{1}, a_{2}^{1}=a_{2}^{2}=u\right)$ is linear in $u$. In other words, for any $a_{1} \in \mathbf{R}_{+}^{2}$, there exist $A \geq 0$ and $B$ such that $V\left(a_{1}, a_{2}^{1}=a_{1}^{2}=u\right)=A u+B$.

In view of the fact that the stage 2 efforts in any $\mathrm{PBE}$ are always symmetric between the two agents by (1), Assumption 2 ensures that the principal's expected payoff is monotonically related to the expected effort in stage $2 .{ }^{17}$ The class of payoff functions $V$ for which Assumption 2 holds includes $V(a)=\sum_{t}\left(a_{t}^{1}+a_{t}^{2}\right), V(a)=\sum_{t} \min \left\{a_{t}^{1}, a_{t}^{2}\right\}$, and more generally, the CES family $V(a)=\sum_{t}\left\{\left(a_{t}^{1}\right)^{m}+\left(a_{t}^{2}\right)^{m}\right\}^{1 / m}(m \in \mathbf{R} \backslash\{0\})$.

Let $v(\sigma, f)$ denote the principal's expected payoff in a PBE $\sigma$ under the feedback policy $f$ :

$$
v(\sigma, f)=E^{\sigma}\left[V\left(\sigma_{1}, \sigma_{2,0}(\tilde{y})\right)\right]
$$

\subsection{Symmetric Equilibrium}

Define

$$
\bar{v}^{*}(f)=\sup \{v(\sigma, f): \sigma \text { is a symmetric pure PBE under } f\}
$$

with the convention that $\bar{v}^{*}(f)=-\infty$ if no such equilibrium exists.

Theorem 4. Suppose that Assumptions 1 and 2 hold and that (*) holds for a sufficiently small $\epsilon>0$. If the marginal cost function $c_{2}^{\prime}$ for stage 2 is convex, then the no-feedback policy maximizes $\bar{v}^{*}$ among all policies.

ProOF: Take any policy $f$ with a symmetric pure PBE $\sigma$. Since $\left(c_{2}^{\prime}\right)^{-1}$ is concave, it follows from Jensen's inequality and the law of iterated expectation that the expected

${ }^{17}$ In the consideration of a symmetric PBE in Section 4.1, we only need the linearity of $V\left(a_{1}, a_{2}^{1}=a_{2}^{2}=u\right)$ in $u$ for $a_{1}$ such that $a_{1}^{1}=a_{1}^{2}$. 
stage 2 effort level in equilibrium satisfies

$$
\begin{aligned}
E^{\sigma}\left[\alpha_{2}\left(\sigma_{1}, \tilde{y}\right)\right] & =E^{\sigma}\left[\left(c_{2}^{\prime}\right)^{-1}\left(E^{\sigma}\left[\phi_{2}\left(\tilde{x}_{1}\right) \mid \tilde{y}\right]\right)\right] \\
& \leq\left(c_{2}^{\prime}\right)^{-1}\left(E^{\sigma}\left[E^{\sigma}\left[\phi_{2}\left(\tilde{x}_{1}\right) \mid \tilde{y}\right]\right]\right) \\
& =\left(c_{2}^{\prime}\right)^{-1}\left(E^{\sigma}\left[\phi_{2}\left(\tilde{x}_{1}\right)\right]\right) \\
& =\left(c_{2}^{\prime}\right)^{-1}\left(\left(\phi_{1} * \phi_{2}\right)(0)\right) \\
& =a_{2}^{*} .
\end{aligned}
$$

Assumption 2 hence implies that for some $A \geq 0$ and $B$,

$$
\begin{aligned}
E^{\sigma}\left[V\left(\sigma_{1}, a_{2}^{1}=a_{2}^{2}=\alpha_{2}\left(\sigma_{1}, \tilde{y}\right)\right)\right] & =E^{\sigma}\left[A \alpha_{2}\left(\sigma_{1}, \tilde{y}\right)+B\right] \\
& =A E^{\sigma}\left[\alpha_{2}\left(\sigma_{1}, \tilde{y}\right)\right]+B \\
& \leq A a_{2}^{*}+B \\
& =V\left(a_{1}^{*}, a_{1}^{*}, a_{2}^{*}, a_{2}^{*}\right) .
\end{aligned}
$$

The desired conclusion follows since the last term equals the principal's expected payoff in the symmetric PBE under the no-feedback policy. //

Theorem 5. Suppose that Assumptions 1 and 2 hold and that $\left(^{*}\right)$ holds for a sufficiently small $\epsilon>0$. If the marginal cost function $c_{2}^{\prime}$ for stage 2 is concave, then the full-feedback policy maximizes $\bar{v}^{*}$ among all policies.

PROOF: As in the proof of Theorem 4, take any policy $f$ which admits a symmetric PBE $\sigma$. Since $\left(c_{2}^{\prime}\right)^{-1}$ is convex, Jensen's inequality now implies that

$$
\begin{aligned}
E^{\sigma}\left[\alpha_{2}\left(\sigma_{1}, \tilde{y}\right)\right] & =E^{\sigma}\left[\left(c_{2}^{\prime}\right)^{-1}\left(E^{\sigma}\left[\phi_{2}\left(\tilde{x}_{1}\right) \mid \tilde{y}\right]\right)\right] \\
& \leq E^{\sigma}\left[E^{\sigma}\left[\left(c_{2}^{\prime}\right)^{-1}\left(\phi_{2}\left(\tilde{x}_{1}\right)\right) \mid \tilde{y}\right]\right] \\
& =E^{\sigma}\left[\left(c_{2}^{\prime}\right)^{-1}\left(\phi_{2}\left(\tilde{x}_{1}\right)\right)\right] .
\end{aligned}
$$

Since $\sigma$ is symmetric, $\sigma_{1}^{1}=\sigma_{1}^{2}=a_{1}^{*}$ by $(2)$, and hence the far right-hand side of the above inequality equals

$$
a_{2}^{* *}=\int_{\mathbf{R}}\left(c_{2}^{\prime}\right)^{-1}\left(\phi_{2}\left(x_{1}\right)\right) \phi_{1}\left(x_{1}\right) d x_{1},
$$

which is the expected stage 2 effort in the symmetric PBE under the full-feedback policy. It therefore follows from Assumption 2 that for some $A \geq 0$ and $B$,

$$
\begin{aligned}
E^{\sigma}\left[V\left(\sigma_{1}, a_{2}^{1}=a_{2}^{2}=\alpha_{2}\left(\sigma_{1}, \tilde{y}\right)\right)\right] & =E^{\sigma}\left[A \alpha_{2}\left(\sigma_{1}, \tilde{y}\right)+B\right] \\
& =A E^{\sigma}\left[\alpha_{2}\left(\sigma_{1}, \tilde{y}\right)\right]+B \\
& \leq A a_{2}^{* *}+B
\end{aligned}
$$


Since the last term equals the principal's expected payoff in the symmetric PBE under the full-feedback policy, the desired conclusion follows. //

The proofs of Theorems 4 and 5 also show that when $c_{2}^{\prime}$ is concave (resp. convex), the no-feedback (resp. full-feedback) policy yields the lowest expected payoff to the principal. On the other hand, when the marginal cost function $c_{2}^{\prime}$ for stage 2 is linear (and hence both concave and convex), the induced effort in either stage is not affected by the feedback policy. The following corollary is an immediate consequence of Theorem 1.

Corollary 6. Suppose that the stage 2 cost function is quadratic: $c_{2}(a)=\frac{1}{2} k a^{2}$ for some $k>0$. Suppose also that Assumption 1 holds and that $\sup _{x \in \mathbf{R}}\left|\phi_{2}^{\prime}(x)\right|<k$. If $\sigma$ is a symmetric pure PBE under any feedback policy $f$, then $\sigma_{1}^{i}=a_{1}^{*}$ and $E^{\sigma}\left[\sigma_{2,0}^{i}(\tilde{y})\right]=$ $\frac{1}{k}\left(\phi_{1} * \phi_{2}\right)(0)$. In particular, the principal's expected payoff $v(\sigma, f)$ is independent of $f$.

\subsection{Asymmetric Equilibrium}

Define now

$$
\bar{v}(f)=\sup \{v(\sigma, f): \sigma \text { is a pure PBE under } f \text { and satisfies }(2)\}
$$

with $\bar{v}(f)=-\infty$ if the corresponding strategy profile does not exist. We will make some additional assumptions in order to evaluate the principal's expected payoff when the agents' effort levels are asymmetric.

Assumption 3: $\left(\phi_{1} * \phi_{2}\right)(0)=\max _{x \in \mathbf{R}}\left(\phi_{1} * \phi_{2}\right)(x)$.

Assumption 4: For any $a=\left(a_{1}^{1}, a_{1}^{2}, a_{2}^{1}, a_{2}^{2}\right) \in \mathbf{R}_{+}^{4}$ such that $a_{1}^{1}<a_{1}^{2}$ and $a_{2}^{1}=a_{2}^{2}$, we have

$$
\frac{c_{1}^{\prime \prime}\left(a_{1}^{1}\right)-2\left(\phi_{1} * \phi_{2}\right)^{\prime}\left(a_{1}^{1}-a_{1}^{2}\right)}{c_{1}^{\prime \prime}\left(a_{1}^{2}\right)+2\left(\phi_{1} * \phi_{2}\right)^{\prime}\left(a_{1}^{1}-a_{1}^{2}\right)}<\frac{\frac{\partial V}{\partial a_{1}^{1}}(a)}{\frac{\partial V}{\partial a_{1}^{2}}(a)} .
$$

Note that the left-hand side of (4) represents the slope of the curve

$$
h\left(a_{1}^{1}, a_{1}^{2}\right) \equiv c_{1}^{\prime}\left(a_{1}^{1}\right)+c_{1}^{\prime}\left(a_{1}^{2}\right)-2\left(\phi_{1} * \phi_{2}\right)\left(a_{1}^{1}-a_{1}^{2}\right)=0
$$

in the $\left(a_{1}^{1}, a_{1}^{2}\right)$-plane, while the right-hand side represents that of the principal's iso-payoff curve. (4) is a single-crossing condition asserting that the iso-profit curve always has a steeper slope than (5). Plainly, Assumption 4 requires that the two agents' efforts be complementary to each other from the point of view of the principal's payoff. To see this, 
suppose that $V$ has the CES form: $V(a)=\sum_{t}\left\{\left(a_{t}^{1}\right)^{m}+\left(a_{t}^{2}\right)^{m}\right\}^{1 / m}(m \in \mathbf{R} \backslash\{0\})$. In this case, the right-hand side of (4) equals $\left(a_{1}^{1} / a_{1}^{2}\right)^{m-1}$. Hence, (4) is easy to satisfy when $m-1$ is negative and large in absolute value. In particular, it will hold for any $c_{1}$ as $m \rightarrow-\infty$, or $V(a)=\sum_{t} \min \left\{a_{t}^{1}, a_{t}^{2}\right\}$. On the other hand, the inequality fails if $m>1$ and $c_{1}^{\prime}$ is concave.

Assumptions 3 and 4 together guarantee that along (5), the principal's payoff is maximized at the symmetric point $\left(a_{1}^{*}, a_{1}^{*}\right)$ (provided that the second-stage efforts are symmetric).

Lemma 7. Suppose that Assumptions 1, 3 and 4 hold and that $\lim _{a \rightarrow \infty} c_{1}^{\prime}(a)>2\left(\phi_{1} *\right.$ $\left.\phi_{2}\right)(0)$ for $i=1,2$. Then for any $\sigma_{1}$ that solves (2) and any $a_{2}$ such that $a_{2}^{1}=a_{2}^{2}$, the principal's payoff function satisfies

$$
V\left(a_{1}^{*}, a_{1}^{*}, a_{2}\right) \geq V\left(\sigma_{1}, a_{2}\right)
$$

Proof: See the Appendix.

TheOREm 8. Suppose that Assumptions 1-4 hold and that (**) holds for a sufficiently small $\epsilon>0$. If the marginal cost function $c_{2}^{\prime}$ for stage 2 is convex, then the no-feedback policy maximizes $\bar{v}(\cdot)$ among all policies.

Proof: See the Appendix. //

Assumption 5: $\phi_{2}$ is unimodal in the sense that $\phi_{2}$ is strictly increasing over $(-\infty, 0)$ and strictly decreasing over $(0, \infty)$.

Note that Assumption 5 implies Assumption 3.

Theorem 9. Suppose that Assumptions 1, 2, 4, and 5 hold, and that (*) holds for a sufficiently small $\epsilon>0$. If the marginal cost function $c_{2}^{\prime}$ for stage 2 is concave, then the full-feedback policy maximizes $\bar{v}(\cdot)$ among all policies.

Proof: See the Appendix. //

As in Section 4.1, we can show that the principal's payoff is independent of a particular feedback policy when the stage-cost function $c_{2}$ is quadratic.

Consider now the feedback policy $f$ that reveals the absolute value of the score: $f\left(x_{1}\right)=\left|x_{1}\right|$ for every $x_{1}$. In other words, this policy reveals the exact size of the lead, but not the identity of the leader. In the two-stage model, this policy is equivalent to the 
full-feedback policy in terms of the induced effort level. To see this, note that if $f$ is as defined above, then

$$
E^{\sigma}\left[\phi_{2}\left(\tilde{x}_{1}\right) \mid \tilde{y}=f\left(x_{1}\right)\right]=\frac{\phi_{2}\left(x_{1}\right)+\phi_{2}\left(-x_{1}\right)}{2}=\phi_{2}\left(x_{1}\right)
$$

by the symmetry of $\phi_{2}$. It follows that the stage 2 effort under $f$ equals that under the fullfeedback policy. It follows that this feedback policy is also optimal under the conditions of Theorems 5 and 9.

\section{T-Stage Tournament}

Suppose now that the tournament game is played over $T$ stages. Let $a_{t}^{i}$ denote agent $i$ 's effort in stage $t$. The score $x_{t}$ in stage $t$ equals $a_{t}^{1}-a_{t}^{2}+\zeta_{t}$, where $\zeta_{t}$ is a random variable with the strictly positive density $\phi_{t}$ over $\mathbf{R}$. We assume that $\phi_{t}$ is symmetric around zero, and twice continuously differentiable. For each $t=1, \ldots, T$, denote by $\omega_{t}=\left(x_{1}, \ldots, x_{t}\right)$ the sequence of scores in stages $1, \ldots, t$, and by $\Delta_{t}$ the aggregate score at the end of stage $t$ :

$$
\Delta_{t}=\sum_{s=1}^{t} x_{s} .
$$

Given $\omega_{t}$ and $s<t$, we also use $\omega_{s}$ to denote the $s$-length truncation of $\omega_{t}$.

The feedback policy $f$ in the $T$-stage tournament is a sequence of (measurable) mappings $f_{1}, \ldots, f_{T-1}$ such that

$$
f_{t}: \mathbf{R}^{t} \rightarrow Y_{t} \quad \text { for } t=1, \ldots, T-1
$$

where $Y_{t}$ is the set of possible announcements after stage $t$. Note that the announcement $y_{t}=f_{t}\left(\omega_{t}\right)$ after stage $t$ may in general depend on all past scores and not just the stage $t$ score. This reflects the fact that a feedback policy in the general $T$-stage model is a significantly more complicated object than that in the two-stage model. For example, the principal may wish to withhold some information for some stages before releasing it with a lag conditional on some subsequent developments, etc.

We say that the feedback policy $f$ is even if $f_{t}\left(\omega_{t}\right)=f_{t}\left(-\omega_{t}\right)$ for every $\omega_{t}$ and $t$. Belonging to this class is the no-feedback policy which has $f_{t}\left(\omega_{t}\right)=f_{t}\left(\omega_{t}^{\prime}\right)$ for every $\omega_{t}, \omega_{t}^{\prime}$ and $t$. Note that the full-feedback policy is not in this class and will be excluded from the following analysis. We will, however, show that there exists an even feedback policy that effectively reveals all the information in a symmetric equilibrium. 
Given any feedback policy $f$, let

$$
Z_{t}(f)=\left\{z_{t}=\left(y_{1}, \ldots, y_{t}\right): y_{s}=f_{s}\left(\omega_{s}\right) \text { for } s=1, \ldots, t \text { for some } \omega_{t}\right\}
$$

be the set of sequences of possible announcements after stages $1, \ldots, t$, and for each $z_{t} \in$ $Z_{t}(f)$, let

$$
X_{t}^{f}\left(z_{t}\right)=\left\{\omega_{t}: f_{s}\left(\omega_{s}\right)=y_{s} \text { for } s=1, \ldots, t\right\}
$$

be the set of sequences of scores compatible with $z_{t}$. In the $T$-period model, we say that $f$ is regular if $X_{t}^{f}\left(z_{t}\right)$ has positive measure or is countable for every $z_{t}$ and $t$.

In order to avoid technical complications arising from boundary problems, we assume that efforts can take negative values.

Assumption 6: For each $t=1, \ldots, T$, the cost function $c_{t}$ in stage $t$ is a mapping from $\mathbf{R}$ to $\mathbf{R}_{+}$and satisfies $c_{t}(0)=c_{t}^{\prime}(0)=0$, and $\inf _{a \in \mathbf{R}} c_{t}^{\prime \prime}(a)>0$.

Under Assumption 6, the cost of negative effort is positive and increases (at an increasing rate) with its absolute size. As seen below, however, only positive efforts are observed along any symmetric equilibrium path.

Agent $i$ 's history after stage $t$, denoted $h_{t}^{i}$, is the sequence of his effort choices $b_{t}^{i} \equiv\left(a_{1}^{i}, \ldots, a_{t}^{i}\right)$ in stages $1, \ldots, t$ along with the sequence of public announcements $z_{t}=\left(y_{1}, \ldots, y_{t}\right)$ after stages $1, \ldots, t$. Agent $i$ 's strategy $\sigma^{i}$ is a sequence $\left(\sigma_{1}, \ldots, \sigma_{T}\right)$, where $\sigma_{t}: \mathbf{R}^{t-1} \times Z_{t-1}(f) \rightarrow \mathbf{R}$ specifies the effort level in stage $t$ as a function of his history $h_{t-1}^{i}$ after stage $t-1$.

Let $\pi_{1}^{i}\left(a_{1}^{i} \mid \sigma\right)$ be agent $i$ 's expected payoff in the entire game when he chooses $a_{1}^{i}$ in stage 1 and then plays according to $\sigma^{i}$ in stages $2, \ldots, T$, and agent $j$ plays according to $\sigma^{j}$ in stages $1, \ldots, T$. Likewise, let $\pi_{t}^{i}\left(a_{t}^{i} \mid \sigma, h_{t-1}^{i}\right)$ be agent $i$ 's expected payoff over stages $t, \ldots, T$ when he chooses $a_{t}^{i}$ in stage $t$ and plays according to $\sigma^{i}$ in stages $t+1, \ldots, T$, his history equals $h_{t-1}^{i}$, and agent $j$ plays according to $\sigma^{j}$. A strategy profile $\sigma$ is a perfect Bayesian equilibrium (PBE) under policy $f$ if for $i=1,2$,

$$
\begin{aligned}
& \pi_{1}^{i}\left(\sigma_{1}^{i} \mid \sigma\right) \geq \pi_{1}^{i}\left(a_{1}^{i} \mid \sigma\right) \text { for every } a_{1}^{i} \in \mathbf{R}, \text { and } \\
& \pi_{t}^{i}\left(\sigma_{t}^{i}\left(h_{t-1}^{i}\right) \mid \sigma, h_{t-1}^{i}\right) \geq \pi_{t}^{i}\left(a_{t}^{i} \mid \sigma, h_{t-1}^{i}\right) \\
& \quad \text { for every } a_{t}^{i} \in \mathbf{R}, h_{t-1}^{i} \in \mathbf{R}^{t-1} \times Z_{t-1}(f), \text { and } t=2, \ldots, T .
\end{aligned}
$$

As before, denote by $\sigma_{t, 0}^{i}\left(z_{t-1}\right)$ agent $i$ 's effort level in stage $t$ along the sequence of public announcements $z_{t-1}$. A PBE is symmetric if the effort levels are symmetric on the path, i.e., $\sigma_{t, 0}^{1}\left(z_{t-1}\right)=\sigma_{t, 0}^{2}\left(z_{t-1}\right)$ for any $z_{t-1} \in Z_{t-1}(f)$ and $t=1, \ldots, T$. 
Let $g_{t-1}\left(\omega_{t-1} \mid z_{t-1}\right)$ denote the density of $\omega_{t-1}=\left(x_{1}, \ldots, x_{t-1}\right)$ conditional on the sequence of public announcements $z_{t-1}=\left(y_{1}, \ldots, y_{t-1}\right)$, provided that both agents play according to a symmetric strategy profile. When $X_{t-1}^{f}\left(z_{t-1}\right)$ has positive measure, it can be written as

$$
g_{t-1}\left(\omega_{t-1} \mid z_{t-1}\right)=\frac{\prod_{s=1}^{t-1} \phi_{s}\left(x_{s}\right)}{\int_{X_{t-1}^{f}\left(z_{t-1}\right)} \prod_{s=1}^{t-1} \phi_{s}\left(x_{s}^{\prime}\right) d \omega_{t-1}^{\prime}}
$$

if $\omega_{t-1} \in X_{t-1}^{f}\left(z_{t-1}\right)$ and $g_{t-1}\left(\omega_{t-1} \mid z_{t-1}\right)=0$ otherwise. The similar expression applies when $X_{t-1}^{f}\left(z_{t-1}\right)$ is countable. Note that $g_{t-1}\left(\omega_{t-1} \mid z_{t-1}\right)$ is independent of any particular symmetric strategy profile $\sigma$.

The following theorem provides sufficient conditions for the existence of a PBE for any even feedback policy in the $T$-stage model and characterizes the effort levels on the equilibrium path.

TheOREM 10. Suppose that Assumption 6 holds. Then there exists $\epsilon>0$ such that if

$$
\begin{aligned}
& \phi_{T}(x),\left|\phi_{T}^{\prime}(x)\right|<\epsilon \text { for any } x \in \mathbf{R}, \text { and } \\
& \left|\phi_{t}^{\prime}(x)\right|,\left|\phi_{t}^{\prime \prime}(x)\right|,\left|\frac{\phi_{t}^{\prime}(x)}{\phi_{t}(x)}\right|,\left|\frac{\phi_{t}^{\prime \prime}(x)}{\phi_{t}(x)}\right|<\epsilon \quad \text { for any } x \in \mathbf{R} \text { and } t=1, \ldots, T-1,
\end{aligned}
$$

then for any even feedback policy $f$, (i) there exists a unique symmetric pure PBE $\sigma=$ $\left(\sigma_{1}, \ldots, \sigma_{T}\right)$ of the $T$-stage tournament, and (ii) the stage $t$ effort on the equilibrium path in any symmetric $P B E$ is given by

$$
\sigma_{t, 0}^{i}\left(z_{t-1}\right)=\left(c_{t}^{\prime}\right)^{-1}\left(E^{\sigma}\left[\phi_{T}\left(\tilde{\Delta}_{T-1}\right) \mid z_{t-1}\right]\right)
$$

for any $z_{t-1} \in Z_{t-1}(f)$ and $t=1, \ldots, T$.

Proof: See the Appendix. //

As is true with the two-stage model in Theorem 2, the existence of an equilibrium in the $T$-stage model requires the noise level to be sufficiently high through condition $(* *)$, and the level $\epsilon$ of noise can be taken independent of the particular feedback policy $f$. Note that $(* *)$ for $T=2$ is more restrictive than $(*)$. For example, the normal distribution, which is allowed under $(*)$, is excluded by $(* *) .{ }^{18}$

$\overline{{ }^{18} \text { On the other hand, }\left({ }^{* *}\right)}$ allows a version of the exponential distribution $f(x)=\gamma e^{-\gamma|x|}$ $(\gamma>0)$. 
We can rewrite (6) more explicitly as

$$
\sigma_{t, 0}^{i}\left(z_{t-1}\right)=\left(c_{t}^{\prime}\right)^{-1}\left(\int_{\mathbf{R}^{T-1}} \phi_{T}\left(\Delta_{T-1}\right) \prod_{s=t}^{T-1} \phi_{s}\left(x_{s}\right) g_{t-1}\left(\omega_{t-1} \mid z_{t-1}\right) d \omega_{T-1}\right)
$$

when $X_{t-1}^{f}\left(z_{t-1}\right)$ has positive measure. This shows that the PBE effort level in the $T$-stage model is a direct extension of that in the two-stage model.

We now study the implication of (6) on the principal's payoff. For this, assume that the principal's payoff function $V: \mathbf{R}^{2 T} \rightarrow \mathbf{R}$ is monotone $(V(\hat{a}) \geq V(a)$ if $\hat{a} \geq a)$ and symmetric $\left(V(a)=V(\hat{a})\right.$ if $a^{1}=\hat{a}^{2}$ and $\left.a^{2}=\hat{a}^{1}\right)$. We also assume that it is monotonically related to the expected efforts when they are symmetric between the agents:

Assumption 7: $V\left(a_{1}^{1}=a_{1}^{2}=u_{1}, a_{2}^{1}=a_{2}^{2}=u_{2}, \ldots, a_{T}^{1}=a_{T}^{2}=u_{T}\right)$ is linear in $\left(u_{2}, \ldots, u_{T}\right)$. In other words, for any $u_{1} \in \mathbf{R}_{+}$, there exist $A_{2}, \ldots, A_{T} \in \mathbf{R}_{+}$and $B \in \mathbf{R}$ such that $V\left(a_{1}^{1}=a_{1}^{2}=u_{1}, a_{2}^{1}=a_{2}^{2}=u_{2}, \ldots, a_{T}^{1}=a_{T}^{2}=u_{T}\right)=\sum_{t=2}^{T} A_{t} u_{t}+B$.

As in Section 4, let $v(\sigma, f)=E^{\sigma}\left[V\left(\sigma_{1}, \sigma_{2,0}\left(\tilde{z}_{1}\right), \ldots, \sigma_{T, 0}\left(\tilde{z}_{T-1}\right)\right)\right]$, and

$$
\bar{v}^{*}(f)=\sup \{v(\sigma, f): \sigma \text { is a symmetric PBE under } f\} .
$$

Theorem 11. Suppose that Assumptions 6 and 7 hold and that (**) holds for a sufficiently small $\epsilon$. If $c_{t}^{\prime}$ is convex for $t=2, \ldots, T$, then the no-feedback policy $f$ maximizes $\bar{v}^{*}$ among all policies.

Proof: Let $a_{t}^{*}$ denote the (deterministic) stage $t$ effort in the symmetric PBE $\sigma$ under the no-feedback policy $f$. Under $f$, we have $X_{t-1}^{f}\left(z_{t-1}\right)=\mathbf{R}_{t-1}$ so that $g_{t-1}\left(\omega_{t-1} \mid z_{t-1}\right)=$ $\prod_{s=1}^{t-1} \phi_{s}\left(x_{s}\right)$. By Theorem 10, hence

$$
\begin{aligned}
c_{t}^{\prime}\left(a_{t}^{*}\right)=E^{\sigma}\left[\phi_{T}\left(\tilde{\Delta}_{T-1}\right)\right] & =\int_{\mathbf{R}^{T-1}} \phi_{T}\left(\Delta_{T-1}\right) \prod_{s=1}^{T-1} \phi_{s}\left(x_{s}\right) d x_{1} \cdots d x_{T-1} \\
& =\left(\phi_{1} * \cdots * \phi_{T}\right)(0),
\end{aligned}
$$

where $\phi_{1} * \cdots * \phi_{T}$ denotes the convolution of $\phi_{1}, \ldots, \phi_{T}$. It follows that $a_{t}^{*}=\left(c_{t}^{\prime}\right)^{-1}\left(\left(\phi_{1} *\right.\right.$ $\left.\left.\cdots * \phi_{T}\right)(0)\right)$. Now let $\sigma^{\prime}$ be a symmetric PBE under any feedback policy $f^{\prime}$. Denote $y_{t}^{\prime}=f_{t}^{\prime}\left(\omega_{t}\right)$ and $z_{t}^{\prime}=\left(y_{1}^{\prime}, \ldots, y_{t}^{\prime}\right)$. Since $\left(c_{t}^{\prime}\right)^{-1}$ is concave, Jensen's inequality implies that 
agent $i$ 's stage $t$ effort under $\left(\sigma^{\prime}\right)^{i}$ satisfies

$$
\begin{aligned}
E^{\sigma^{\prime}}\left[\left(\sigma^{\prime}\right)_{t}^{i}\left(z_{t-1}^{\prime}\right)\right] & =E^{\sigma^{\prime}}\left[\left(c_{t}^{\prime}\right)^{-1}\left(E^{\sigma^{\prime}}\left[\phi_{T}\left(\tilde{\Delta}_{T-1}\right) \mid \tilde{z}_{t-1}^{\prime}\right]\right)\right] \\
& \leq\left(c_{t}^{\prime}\right)^{-1}\left(E^{\sigma^{\prime}}\left[E^{\sigma^{\prime}}\left[\phi_{T}\left(\tilde{\Delta}_{T-1}\right) \mid \tilde{z}_{t-1}^{\prime}\right]\right]\right) \\
& =\left(c_{t}^{\prime}\right)^{-1}\left(E^{\sigma^{\prime}}\left[\phi_{T}\left(\tilde{\Delta}_{T-1}\right)\right]\right) \\
& =\left(c_{t}^{\prime}\right)^{-1}\left(\left(\phi_{1} * \cdots * \phi_{T}\right)(0)\right)=a_{t}^{*}
\end{aligned}
$$

This completes the proof. //

When $c_{t}^{\prime}$ is concave, we expect the optimal policy to be the one that reveals the most information. As mentioned earlier, however, the full-feedback policy does not admit a symmetric PBE when $T \geq 3$. We instead consider the even policy $f$ that reveals the absolute value of the aggregate score at the end of each stage:

$$
f_{t}\left(\omega_{t}\right)=\left|\Delta_{t}\right| \quad t=1, \ldots, T-1
$$

Note that the discussion at the end of Section 4 shows that such a policy induces the same effort level as the full-feedback policy in the two-stage model. The next theorem shows that $f$ given in $(7)$ is indeed optimal.

TheOrem 12. Suppose that Assumptions 6 and 7 hold and that (**) holds for a sufficiently small $\epsilon>0$. If $c_{t}^{\prime}$ is concave for $t=2, \ldots, T$, then the feedback policy $f$ in (7) that reveals the absolute value of the aggregate score after every stage maximizes $\bar{v}^{*}$ among all policies.

Proof: See the Appendix. //

\section{Appendix}

When the distinction is necessary, equations in the Appendix assume for simplicity that the set of compatible scores $f^{-1}(y)$ or $X_{t-1}^{f}\left(z_{t-1}\right)$ has positive measure. When it is countable, any integral with respect to the conditional density should be replaced by the summation over $f^{-1}(y)$ or $X_{t-1}^{f}\left(z_{t-1}\right)$.

Proof of Theorem 1: Fix any PBE $\sigma$. With slight abuse of notation, let $g_{1}^{\sigma}\left(x_{1} \mid a_{1}^{i}, y\right)$ denote the density of $x_{1}$ conditional on the public announcement $y$ when the stage 1 actions are $a_{1}^{i}$ for agent $i$ and $\sigma_{1}^{j}$ for agent $j$ : For example, when $f^{-1}(y)$ has positive measure, we 
have for $x_{1} \in f^{-1}(y)$,

$$
\begin{aligned}
& g_{1}^{\sigma}\left(x_{1} \mid a_{1}^{1}, y\right)=\frac{\phi_{1}\left(x_{1}-a_{1}^{1}+\sigma_{1}^{2}\right)}{\int_{f^{-1}(y)} \phi_{1}\left(x_{1}^{\prime}-a_{1}^{1}+\sigma_{1}^{2}\right) d x_{1}^{\prime}}, \quad \text { and } \\
& g_{1}^{\sigma}\left(x_{1} \mid a_{1}^{2}, y\right)=\frac{\phi_{1}\left(x_{1}-\sigma_{1}^{1}+a_{1}^{2}\right)}{\int_{f^{-1}(y)} \phi_{1}\left(x_{1}^{\prime}-\sigma_{1}^{1}+a_{1}^{2}\right) d x_{1}^{\prime}} .
\end{aligned}
$$

Note in particular that $g_{1}^{\sigma}\left(x_{1} \mid \sigma_{1}^{1}, y\right)=g_{1}^{\sigma}\left(x_{1} \mid \sigma_{1}^{2}, y\right)=g_{1}^{\sigma}\left(x_{1} \mid y\right)$. Recall that $\pi_{2}^{i}\left(a_{2}^{i} \mid\right.$ $\left.\sigma, a_{1}^{i}, y\right)$ represents agent $i$ 's expected payoff in stage 2 when he chooses $a_{2}^{i}$ in stage 2 , his history after stage 1 is $h_{1}^{i}=\left(a_{1}^{i}, y\right)$, and agent $j$ plays according to the equilibrium strategy $\sigma^{j}$. For simplicity, write $\pi_{2}^{i}\left(a_{2}^{i} \mid a_{1}^{i}, y\right)$ for $\pi_{2}^{i}\left(a_{2}^{i} \mid \sigma, a_{1}^{i}, y\right)$. It can be seen that $\pi_{2}^{i}\left(a_{2}^{i} \mid a_{i}^{i}, y\right)$ is written as

$$
\pi_{2}^{1}\left(a_{2}^{1} \mid a_{1}^{1}, y\right)=\int_{\mathbf{R}} \Phi_{2}\left(a_{2}^{1}-\sigma_{2,0}^{2}(y)+x_{1}\right) g_{1}^{\sigma}\left(x_{1} \mid a_{1}^{1}, y\right) d x_{1}-c_{2}\left(a_{2}^{1}\right)
$$

for agent 1 , and

$$
\pi_{2}^{2}\left(a_{2}^{2} \mid a_{1}^{2}, y\right)=\int_{\mathbf{R}} \Phi_{2}\left(-\sigma_{2,0}^{1}(y)+a_{2}^{2}-x_{1}\right) g_{1}^{\sigma}\left(x_{1} \mid a_{1}^{2}, y\right) d x_{1}-c_{2}\left(a_{2}^{2}\right)
$$

for agent 2. Differentiating $\pi_{2}^{1}$ with respect to $a_{2}^{1}$, we obtain

$$
\frac{\partial \pi_{2}^{1}}{\partial a_{2}^{1}}\left(a_{2}^{1} \mid a_{1}^{1}, y\right)=\int_{\mathbf{R}} \phi_{2}\left(a_{2}^{1}-\sigma_{2,0}^{2}(y)+x_{1}\right) g_{1}^{\sigma}\left(x_{1} \mid a_{1}^{1}, y\right) d x_{1}-c_{2}^{\prime}\left(a_{2}^{1}\right)
$$

Since $c_{2}^{\prime}(0)=0$ implies $\frac{\partial \pi_{2}^{1}}{\partial a_{2}^{1}}\left(0 \mid a_{1}^{1}, y\right)>0$, the equilibrium action $\sigma_{2}^{1}\left(a_{1}^{1}, y\right)$ (if any) must satisfy

$$
\frac{\partial \pi_{2}^{1}}{\partial a_{2}^{1}}\left(\sigma_{2}^{1}\left(a_{1}^{1}, y\right) \mid a_{1}^{1}, y\right)=0
$$

or equivalently,

$$
c_{2}^{\prime}\left(\sigma_{2}^{1}\left(a_{1}^{1}, y\right)\right)=\int_{\mathbf{R}} \phi_{2}\left(\sigma_{2}^{1}\left(a_{1}^{1}, y\right)-\sigma_{2,0}^{2}(y)+\tilde{x}_{1}\right) g_{1}^{\sigma}\left(x_{1} \mid a_{1}^{1}, y\right) d x_{1}
$$

for every $a_{1}^{1}$. By our assumption that $\inf _{a \in \mathbf{R}_{+}} c_{2}^{\prime \prime}(a)>\sup _{x \in \mathbf{R}}\left|\phi_{2}^{\prime}(x)\right|$, we have $\frac{\partial^{2} \pi_{2}^{1}}{\partial\left(a_{2}^{1}\right)^{2}}\left(a_{2}^{1} \mid\right.$ $\left.a_{1}^{1}, y\right)<0$, which shows that $\sigma_{2}^{1}(\cdot, y)$ is differentiable as a function of $a_{1}^{1}$ by the implicit function theorem. For agent 2, we have

$$
c_{2}^{\prime}\left(\sigma_{2}^{2}\left(a_{1}^{2}, y\right)\right)=\int_{\mathbf{R}} \phi_{2}\left(-\sigma_{2,0}^{1}(y)+\sigma_{2}^{2}\left(a_{1}^{2}, y\right)-\tilde{x}_{1}\right) g_{1}^{\sigma}\left(x_{1} \mid a_{1}^{2}, y\right) d x_{1}
$$


for every $a_{1}^{2}$. Noting $\sigma_{2}^{i}\left(\sigma_{1}^{i}, y\right)=\sigma_{2,0}^{i}(y)$ and $g_{1}^{\sigma}\left(x_{1} \mid \sigma_{1}^{i}, y\right)=g_{1}^{\sigma}\left(x_{1} \mid y\right)$, we substitute $a_{1}^{i}=\sigma_{1}^{i}(i=1,2)$ into (a3) and (a4) to obtain

$$
\sigma_{2,0}^{1}(y)=\sigma_{2,0}^{2}(y)=\alpha_{2}(y) \equiv\left(c_{2}^{\prime}\right)^{-1}\left(E^{\sigma}\left[\phi_{2}\left(\tilde{x}_{1}\right) \mid y\right]\right)
$$

Now let $\pi_{1}^{i}\left(a_{1}^{i}\right)=\pi_{1}^{i}\left(a_{1}^{i} \mid \sigma\right)$ be agent $i$ 's (overall) expected payoff when he takes $a_{1}^{i}$ in stage 1 and $\sigma_{2}^{i}\left(a_{1}^{i}, y\right)$ in stage 2 , while agent $j$ plays according to his equilibrium strategy $\sigma^{j}$. For $i=1$, we have

$$
\begin{aligned}
\pi_{1}^{1}\left(a_{1}^{1}\right) & =-c_{1}\left(a_{1}^{1}\right) \\
& +\int_{\mathbf{R}}\left\{\Phi_{2}\left(\sigma_{2}^{1}\left(a_{1}^{1}, f\left(x_{1}\right)\right)-\sigma_{2,0}^{2}\left(f\left(x_{1}\right)\right)+x_{1}\right)-c_{2}\left(\sigma_{2}^{1}\left(a_{1}^{1}, f\left(x_{1}\right)\right)\right)\right\} \\
& \times \phi_{1}\left(x_{1}-a_{1}^{1}+\sigma_{1}^{2}\right) d x_{1} .
\end{aligned}
$$

In view of the differentiability of $\sigma_{2}^{1}$ in $a_{1}^{1}$ noted above, we can differentiate $\pi_{1}^{1}$ using the envelope theorem to obtain

$$
\begin{aligned}
\left(\pi_{1}^{1}\right)^{\prime}\left(a_{1}^{1}\right) & =-\int_{\mathbf{R}} \Phi_{2}\left(\sigma_{2}^{1}\left(a_{1}^{1}, f\left(x_{1}\right)\right)-\sigma_{2,0}^{2}\left(f\left(x_{1}\right)\right)+x_{1}\right) \phi_{1}^{\prime}\left(x_{1}-a_{1}^{1}+\sigma_{1}^{2}\right) d x_{1} \\
& +\int_{\mathbf{R}} c_{2}\left(\sigma_{2}^{1}\left(a_{1}^{1}, f\left(x_{1}\right)\right)\right) \phi_{1}^{\prime}\left(x_{1}-a_{1}^{1}+\sigma_{1}^{2}\right) d x_{1}-c_{1}^{\prime}\left(a_{1}^{1}\right) .
\end{aligned}
$$

If the equilibrium stage 1 action $a_{1}^{1}=\sigma_{1}^{1}$ is strictly positive, the FOC $\left(\pi_{1}^{1}\right)^{\prime}\left(\sigma_{1}^{1}\right)=0$ must hold. Since $\sigma_{2,0}^{1}(y)=\sigma_{2,0}^{2}(y)$ for any $y \in Y$ by (a5), this FOC is equivalent to

$$
c_{1}^{\prime}\left(\sigma_{1}^{1}\right)=-\int_{\mathbf{R}} \Phi_{2}\left(x_{1}\right) \phi_{1}^{\prime}\left(x_{1}-\sigma_{1}^{1}+\sigma_{1}^{2}\right) d x_{1}+\int_{\mathbf{R}} c_{2}\left(\alpha_{2}\left(\sigma_{1}, f\left(x_{1}\right)\right)\right) \phi_{1}^{\prime}\left(x_{1}-\sigma_{1}^{1}+\sigma_{1}^{2}\right) d x_{1} .
$$

Changing variables of the first integral, and then integrating it by parts, we see that this is equivalent to the first line of (2). The symmetric argument shows that the second line of (2) is equivalent to the FOC for agent 2.

Proof of Theorem 2: Choose $\epsilon>0$ such that $\epsilon<\kappa / 2$, where

$$
\kappa=\min \left\{1, \inf _{a \in \mathbf{R}_{+}} c_{1}^{\prime \prime}(a), \inf _{a \in \mathbf{R}_{+}} c_{2}^{\prime \prime}(a), \lim _{a \rightarrow \infty} c_{2}^{\prime}(a)\right\}
$$

Suppose that $\sigma_{1}=\left(\sigma_{1}^{1}, \sigma_{1}^{2}\right)$ solves $(2)$ and consider the equations

$$
\begin{aligned}
& \varphi_{2}^{1}\left(a_{2}^{1} \mid a_{1}^{1}, y\right) \equiv \int_{\mathbf{R}} \phi_{2}\left(a_{2}^{1}-\alpha_{2}\left(\sigma_{1}, y\right)+x_{1}\right) g_{1}^{\sigma}\left(x_{1} \mid a_{1}^{1}, y\right) d x_{1}-c_{2}^{\prime}\left(a_{2}^{1}\right)=0, \quad \text { and } \\
& \varphi_{2}^{2}\left(a_{2}^{2} \mid a_{1}^{2}, y\right) \equiv \int_{\mathbf{R}} \phi_{2}\left(\alpha_{2}\left(\sigma_{1}, y\right)-a_{2}^{2}+x_{1}\right) g_{1}^{\sigma}\left(x_{1} \mid a_{1}^{2}, y\right) d x_{1}-c_{2}^{\prime}\left(a_{2}^{2}\right)=0
\end{aligned}
$$


for each $a_{1}^{1}, a_{1}^{2} \in \mathbf{R}$, and $y \in Y$. We have $\varphi_{2}^{1}\left(0 \mid a_{1}^{1}, y\right)>0$ by $c_{2}^{\prime}(0)=0$ and $\phi_{2}>0$, and $\varphi_{2}^{1}\left(a_{2}^{1} \mid a_{1}^{1}, y\right)<0$ for $a_{2}^{1}$ large enough since $\lim _{a \rightarrow \infty} c_{2}^{\prime}(a)>\epsilon>\sup _{x \in \mathbf{R}} \phi_{2}(x)$. Furthermore, it follows from $\inf _{a \in \mathbf{R}} c_{2}^{\prime \prime}(a)>\epsilon>\sup _{x \in \mathbf{R}}\left|\phi_{2}^{\prime}(x)\right|$ that

$$
\frac{\partial \varphi_{2}^{1}}{\partial a_{2}^{1}}\left(a_{2}^{1} \mid a_{1}^{1}, y\right)=-c_{2}^{\prime \prime}\left(a_{2}^{1}\right)+\int_{\mathbf{R}} \phi_{2}^{\prime}\left(a_{2}^{1}-\sigma_{2,0}^{2}(y)+x_{1}\right) g_{1}^{\sigma}\left(x_{1} \mid a_{1}^{1}, y\right) d x_{1}<0 .
$$

Hence, there exists a unique solution to $\varphi_{2}^{1}\left(a_{2}^{1} \mid a_{1}^{1}, y\right)=0$, and we define $a_{2}^{1}=\sigma_{2}^{1}\left(a_{1}^{1}, y\right)>0$ to be this solution. In the same manner, $\sigma_{2}^{2}\left(a_{1}^{2} \mid a_{1}^{2}, y\right)$ is defined to be the unique solution to $\varphi_{2}^{2}\left(a_{2}^{2} \mid a_{1}^{2}, y\right)=0$. Note now that when $a_{1}^{1}=\sigma_{1}^{1}$ and $a_{1}^{2}=\sigma_{1}^{2}, \sigma_{2}^{1}\left(\sigma_{1}^{1}, y\right)=\sigma_{2}^{2}\left(\sigma_{1}^{2}, y\right)=$ $\alpha_{2}\left(\sigma_{1}, y\right)$ solves the two equations. This implies that $\varphi_{2}^{1}\left(a_{2}^{1} \mid a_{1}^{1}, y\right)=0$ is equivalent to the FOC $\frac{\partial \pi_{2}^{1}}{\partial a_{2}^{1}}\left(a_{2}^{1} \mid a_{1}^{1}, y\right)=0$ ((a3) in the proof of Theorem 1) of agent $i$ 's stage 2 payoff maximization problem. To see that it does maximize his payoff, it suffices to note that

$$
\begin{aligned}
\frac{\partial^{2} \pi_{2}^{1}}{\partial\left(a_{2}^{1}\right)^{2}}\left(a_{2}^{1} \mid a_{1}^{1}, y\right) & =-c_{2}^{\prime \prime}\left(a_{2}^{1}\right)+\int_{\mathbf{R}} \phi_{2}^{\prime}\left(a_{2}^{1}-\sigma_{2,0}^{2}(y)+x_{1}\right) g_{1}^{\sigma}\left(x_{1} \mid a_{1}^{1}, y\right) d x_{1} \\
& <-\kappa+\epsilon<0 .
\end{aligned}
$$

The same observation holds for agent 2. As in the proof of Theorem 1 , denote by $\pi_{1}^{i}\left(a_{1}^{i}\right)$ agent $i$ 's overall payoff when he takes action $a_{1}^{i}$ in stage 1 and chooses $\sigma_{2}^{i}\left(a_{1}^{i}, y\right)$ in stage 2 , and agent $j$ takes action $\sigma_{1}^{j}$ in stage 1 and chooses $\sigma_{2}^{j}\left(\sigma_{1}^{j}, y\right)$ in stage 2. Define

$$
\begin{aligned}
& \varphi_{1}^{1}\left(a_{1}^{1}\right)=-c_{1}^{\prime}\left(a_{1}^{1}\right)+\left(\phi_{1} * \phi_{2}\right)\left(a_{1}^{1}-\sigma_{1}^{2}\right)+\int_{\mathbf{R}} c_{2}\left(\alpha_{2}\left(a_{1}^{1}, \sigma_{1}^{2}, f\left(x_{1}\right)\right)\right) \phi_{1}^{\prime}\left(x_{1}-a_{1}^{1}+\sigma_{1}^{2}\right) d x_{1}, \\
& \varphi_{1}^{2}\left(a_{1}^{2}\right)=-c_{1}^{\prime}\left(a_{1}^{2}\right)+\left(\phi_{1} * \phi_{2}\right)\left(\sigma_{1}^{1}-a_{1}^{2}\right)-\int_{\mathbf{R}} c_{2}\left(\alpha_{2}\left(\sigma_{1}^{1}, a_{1}^{2}, f\left(x_{1}\right)\right)\right) \phi_{1}^{\prime}\left(x_{1}-\sigma_{1}^{1}+a_{1}^{2}\right) d x_{1} .
\end{aligned}
$$

By assumption, $a_{1}^{i}=\sigma_{1}^{i}$ solves $\varphi_{1}^{i}\left(a_{1}^{i}\right)=0$. Furthermore, $\varphi_{1}^{i}\left(a_{1}^{i}\right)=\left(\pi_{1}^{i}\right)^{\prime}\left(a_{1}^{i}\right)$ as seen in the proof of Theorem 1 so that $\sigma_{1}^{i}$ is a solution to the FOC of agent $i$ 's payoff maximization problem. In what follows, We will show $\left(\varphi_{1}^{i}\right)^{\prime}=\left(\pi_{1}^{i}\right)^{\prime \prime}<0$ and hence $\sigma_{1}^{i}$ is indeed the maximizer of $\pi_{1}^{1}$.

Since $\sigma_{2}^{1}$ is differentiable with respect to $a_{1}^{1}$, we can differentiate (a7) to obtain

$$
\begin{aligned}
& \left(\varphi_{1}^{1}\right)^{\prime}\left(a_{1}^{1}\right)=-c_{1}^{\prime \prime}\left(a_{1}^{1}\right) \\
& -\int_{\mathbf{R}}\left\{\phi_{2}\left(\sigma_{2}^{1}\left(a_{1}^{1}, f\left(x_{1}\right)\right)-\sigma_{2,0}^{2}\left(f\left(x_{1}\right)\right)+x_{1}\right)-c_{2}^{\prime}\left(\sigma_{2}^{1}\left(a_{1}^{1}, f\left(x_{1}\right)\right)\right)\right\} \\
& \times \frac{\partial \sigma_{2}^{1}}{\partial a_{1}^{1}}\left(a_{1}^{1}, f\left(x_{1}\right)\right) \phi_{1}^{\prime}\left(x_{1}-a_{1}^{1}+\sigma_{1}^{2}\right) d x_{1} \\
& +\int_{\mathbf{R}}\left\{\Phi_{2}\left(\sigma_{2}^{1}\left(a_{1}^{1}, f\left(x_{1}\right)\right)-\sigma_{2,0}^{2}\left(f\left(x_{1}\right)\right)+x_{1}\right)-c_{2}\left(\sigma_{2}^{1}\left(a_{1}^{1}, f\left(x_{1}\right)\right)\right)\right\} \phi_{1}^{\prime \prime}\left(x_{1}-a_{1}^{1}+\sigma_{1}^{2}\right) d x_{1} .
\end{aligned}
$$


We have $c_{2}^{\prime}\left(\sigma_{2}^{1}\right) \leq \epsilon$ by $(\mathrm{a} 4)$ and $c_{2}\left(\sigma_{2}^{1}\right) \leq 1$ by the above observation that $\sigma_{2}^{1}$ maximizes $\pi_{2}^{1}$. Hence,

$$
\left|\phi_{2}\left(\sigma_{2}^{1}-\sigma_{2,0}^{2}+x_{1}\right)-c_{2}^{\prime}\left(\sigma_{2}^{1}\right)\right| \leq \epsilon
$$

and

$$
\left|\Phi_{2}\left(\sigma_{2}^{1}-\sigma_{2,0}^{2}+x_{1}\right)-c_{2}\left(\sigma_{2}^{1}\right)\right| \leq 1
$$

It follows that

$$
\begin{aligned}
\left(\varphi_{1}^{1}\right)^{\prime}\left(a_{1}^{1}\right) & \leq-c_{1}^{\prime \prime}\left(a_{1}^{1}\right)+\epsilon \int_{\mathbf{R}}\left|\frac{\partial \sigma_{2}^{1}}{\partial a_{1}^{1}}\left(a_{1}^{1}, f\left(x_{1}\right)\right)\right|\left|\phi_{1}^{\prime}\left(x_{1}-a_{1}^{1}+\sigma_{1}^{2}\right)\right| d x_{1} \\
& +\int_{\mathbf{R}}\left|\phi_{1}^{\prime \prime}\left(x_{1}-a_{1}^{1}+\sigma_{1}^{2}\right)\right| d x_{1} .
\end{aligned}
$$

Now take $y \in Y$ such that $f^{-1}(y)$ has positive measure. Then for $x_{1} \in f^{-1}(y)$,

$$
\begin{aligned}
\frac{\partial g_{1}^{\sigma}}{\partial a_{1}^{1}}\left(x_{1} \mid a_{1}^{1}, y\right) & =\frac{-\phi_{1}^{\prime}\left(x_{1}-a_{1}^{1}+\sigma_{1}^{2}\right)}{\int_{f^{-1}(y)} \phi_{1}\left(\hat{x}_{1}-a_{1}^{1}+\sigma_{1}^{2}\right) d \hat{x}_{1}} \\
& +\frac{\phi_{1}\left(x_{1}-a_{1}^{1}+\sigma_{1}^{2}\right) \int_{f^{-1}(y)} \phi_{1}^{\prime}\left(\hat{x}_{1}-a_{1}^{1}+\sigma_{1}^{2}\right) d \hat{x}_{1}}{\left\{\int_{f^{-1}(y)} \phi_{1}\left(\hat{x}_{1}-a_{1}^{1}+\sigma_{1}^{2}\right) d \hat{x}_{1}\right\}^{2}}
\end{aligned}
$$

and hence

$$
\begin{aligned}
\int_{\mathbf{R}}\left|\frac{\partial g_{1}^{\sigma}}{\partial a_{1}^{1}}\left(x_{1} \mid a_{1}^{1}, y\right)\right| d x_{1} & \leq 2 \frac{\int_{f^{-1}(y)}\left|\phi_{1}^{\prime}\left(x_{1}-a_{1}^{1}+\sigma_{1}^{2}\right)\right| d x_{1}}{\int_{f^{-1}(y)} \phi_{1}\left(x_{1}-a_{1}^{1}+\sigma_{1}^{2}\right) d x_{1}} \\
& =2 E\left[q\left(x_{1}\right) \mid a_{1}^{1}, \sigma_{1}^{2}, y\right]
\end{aligned}
$$

where

$$
q\left(x_{1}\right)=\frac{\left|\phi_{1}^{\prime}\left(x_{1}-a_{1}^{1}+\sigma_{1}^{2}\right)\right|}{\phi_{1}\left(x_{1}-a_{1}^{1}+\sigma_{1}^{2}\right)}
$$

On the other hand,

$$
\frac{\partial \varphi_{2}^{1}}{\partial a_{1}^{1}}\left(a_{2}^{1} \mid a_{1}^{1}, y\right)=\int_{\mathbf{R}} \phi_{2}\left(\sigma_{2}^{1}-\sigma_{2,0}^{2}+x_{1}\right) \frac{\partial g_{1}^{\sigma}}{\partial a_{1}^{1}}\left(x_{1} \mid a_{1}^{1}, y\right) d x_{1}
$$

so that

$$
\left|\frac{\partial \varphi_{2}^{1}}{\partial a_{1}^{1}}\left(a_{2}^{1} \mid a_{1}^{1}, y\right)\right|<\epsilon \int_{\mathbf{R}}\left|\frac{\partial g_{1}^{\sigma}}{\partial a_{1}^{1}}\left(x_{1} \mid a_{1}^{1}, y\right)\right| d x_{1} \leq 2 \epsilon E\left[q\left(x_{1}\right) \mid a_{1}^{1}, \sigma_{1}^{2}, y\right] .
$$


Therefore,

$$
\begin{aligned}
& \frac{1}{2 \epsilon} \int_{\mathbf{R}}\left|\frac{\partial \varphi_{2}^{1}}{\partial a_{1}^{1}}\right|\left|\phi_{1}^{\prime}\left(x_{1}-a_{1}^{1}+\sigma_{1}^{2}\right)\right| d x_{1} \\
& \leq \int_{\mathbf{R}} E\left[q\left(\tilde{x}_{1}\right) \mid a_{1}^{1}, \sigma_{1}^{2}, \tilde{y}=f\left(x_{1}\right)\right] q\left(x_{1}\right) \phi_{1}\left(x_{1}-a_{1}^{1}+\sigma_{1}^{2}\right) d x_{1} \\
& =E\left[E\left[q\left(\tilde{x}_{1}\right) \mid a_{1}^{1}, \sigma_{1}^{2}, \tilde{y}\right] q\left(\tilde{x}_{1}\right) \mid a_{1}^{1}, \sigma_{1}^{2}\right] \\
& \leq E\left[E\left[q\left(\tilde{x}_{1}\right) \mid a_{1}^{1}, \sigma_{1}^{2}, \tilde{y}\right]^{2} \mid a_{1}^{1}, \sigma_{1}^{2}\right]^{1 / 2} E\left[q\left(\tilde{x}_{1}\right)^{2} \mid a_{1}^{1}, \sigma_{1}^{2}\right]^{1 / 2} \\
& \leq E\left[E\left[q\left(\tilde{x}_{1}\right)^{2} \mid a_{1}^{1}, \sigma_{1}^{2}, \tilde{y}\right] \mid a_{1}^{1}, \sigma_{1}^{2}\right]^{1 / 2} E\left[q\left(\tilde{x}_{1}\right)^{2} \mid a_{1}^{1}, \sigma_{1}^{2}\right]^{1 / 2} \\
& =E\left[q\left(\tilde{x}_{1}\right)^{2} \mid a_{1}^{1}, \sigma_{1}^{2}\right]=\int_{\mathbf{R}}\left|\frac{\phi_{1}^{\prime}\left(x_{1}\right)}{\phi_{1}\left(x_{1}\right)}\right|^{2} \phi_{1}\left(x_{1}\right) d x_{1}<\epsilon,
\end{aligned}
$$

where the fourth line follows from Schwartz' inequality and the fifth line from Jensen's inequality. Using the implicit function theorem, we see that the second term on the righthand side of (a8) can be evaluated as:

$$
\begin{aligned}
& \epsilon \int_{\mathbf{R}}\left|\frac{\partial \sigma_{2}^{1}}{\partial a_{1}^{1}}\left(a_{1}^{1}, f\left(x_{1}\right)\right)\right|\left|\phi_{1}^{\prime}\left(x_{1}-a_{1}^{1}+\sigma_{1}^{2}\right)\right| d x_{1} \\
& =\epsilon \int_{\mathbf{R}} \frac{\left|\frac{\partial \varphi_{2}^{1}}{\partial a_{1}^{1}}\left(\sigma_{2}^{1}\left(a_{1}^{1}, f\left(x_{1}\right)\right) \mid a_{1}^{1}, y\right)\right|}{\left|\frac{\partial \varphi_{2}^{1}}{\partial a_{2}^{1}}\left(\sigma_{2}^{1}\left(a_{1}^{1}, f\left(x_{1}\right)\right) \mid a_{1}^{1}, y\right)\right|}\left|\phi_{1}^{\prime}\left(x_{1}-a_{1}^{1}+\sigma_{1}^{2}\right)\right| d x_{1} \\
& \leq \frac{\epsilon}{\kappa-\epsilon} \int_{\mathbf{R}}\left|\frac{\partial \varphi_{2}^{1}}{\partial a_{1}^{1}}\left(\sigma_{2}^{1}\left(a_{1}^{1}, f\left(x_{1}\right)\right) \mid a_{1}^{1}, f\left(x_{1}\right)\right)\right|\left|\phi_{1}^{\prime}\left(x_{1}-a_{1}^{1}+\sigma_{1}^{2}\right)\right| d x_{1} \\
& \leq \frac{2 \epsilon^{3}}{\kappa-\epsilon}
\end{aligned}
$$

Hence,

$$
\left(\varphi_{1}^{1}\right)^{\prime}\left(a_{1}^{1}\right) \leq-\kappa+\frac{2 \epsilon^{3}}{\kappa-\epsilon}+\epsilon<0
$$

This proves the claim. //

Proof of Theorem 3: Suppose that $\sigma_{1}^{1}=\sigma_{1}^{2}$. We first show that $\alpha_{2}\left(\sigma_{1}, f\left(x_{1}\right)\right)=$ $\alpha_{2}\left(f\left(-x_{1}\right)\right)$ for any $x_{1}$. This would hold trivially if $f$ is even since then $f\left(x_{1}\right)=f\left(-x_{1}\right)$. If $f$ is odd, then $g_{1}^{\sigma}\left(x_{1} \mid y\right)=g_{1}^{\sigma}\left(-x_{1} \mid-y\right)$, and hence the symmetry of $\phi_{2}$ implies that

$$
\begin{aligned}
\alpha_{2}\left(\sigma_{1}, y\right) & =\left(c_{2}^{\prime}\right)^{-1}\left(\int_{\mathbf{R}} \phi_{2}\left(x_{1}\right) g_{1}^{\sigma}\left(x_{1} \mid y\right) d x_{1}\right) \\
& =\left(c_{2}^{\prime}\right)^{-1}\left(\int_{\mathbf{R}} \phi_{2}\left(-x_{1}\right) g_{1}^{\sigma}\left(-x_{1} \mid-y\right) d x_{1}\right) \\
& =\alpha_{2}\left(\sigma_{1},-y\right) .
\end{aligned}
$$


It follows that $\alpha_{2}\left(f\left(-x_{1}\right)\right)=\alpha_{2}\left(\sigma_{1},-f\left(x_{1}\right)\right)=\alpha_{2}\left(\sigma_{1}, f\left(x_{1}\right)\right)$. With this equality, $\sigma_{1}^{1}=$ $\sigma_{1}^{2}=a_{1}^{*}$ solves $(2)$ since

$$
\begin{aligned}
& \int_{\mathbf{R}} c_{2}\left(\alpha_{2}\left(\sigma_{1}, f\left(x_{1}\right)\right)\right) \phi_{1}^{\prime}\left(x_{1}\right) d x_{1} \\
& =\int_{0}^{\infty} c_{2}\left(\alpha_{2}\left(\sigma_{1}, f\left(x_{1}\right)\right)\right) \phi_{1}^{\prime}\left(x_{1}\right) d x_{1}+\int_{-\infty}^{0} c_{2}\left(\alpha_{2}\left(\sigma_{1}, f\left(x_{1}\right)\right)\right) \phi_{1}^{\prime}\left(x_{1}\right) d x_{1} \\
& =\int_{0}^{\infty} c_{2}\left(\alpha_{2}\left(\sigma_{1}, f\left(x_{1}\right)\right)\right) \phi_{1}^{\prime}\left(x_{1}\right) d x_{1}-\int_{0}^{\infty} c_{2}\left(\alpha_{2}\left(f\left(-x_{1}\right)\right)\right) \phi_{1}^{\prime}\left(x_{1}\right) d x_{1} \\
& =0 .
\end{aligned}
$$

This completes the proof. //

Proof of Lemma 7: Fix $a_{2} \in \mathbf{R}_{+}^{2}$ such that $a_{2}^{1}=a_{2}^{2}$. Since $h$ is continuous, the inverse image $h^{-1}(\{0\})$ is closed. Furthermore, it is non-empty since $\left(a_{1}^{*}, a_{1}^{*}\right) \in h^{-1}(\{0\})$ and bounded since for any $a_{1}^{j} \in \mathbf{R}_{+}, h\left(a_{1}^{i}, a_{1}^{j}\right)>0$ for $a_{1}^{i}>\left(c_{1}^{\prime}\right)^{-1}\left(2\left(\phi_{1} * \phi_{2}\right)(0)\right)$ by assumption. It follows that the continuous function $V\left(\cdot, a_{2}\right)$ on the compact set $h^{-1}(\{0\})=\left\{a_{1} \in \mathbf{R}_{+}^{2}\right.$ : $\left.h\left(a_{1}\right)=0\right\}$ achieves a maximum. Let $\bar{a}_{1}=\left(\bar{a}_{1}^{1}, \bar{a}_{1}^{2}\right) \in h^{-1}(\{0\})$ be any maximizer of $V\left(\cdot, a_{2}\right)$ in $h^{-1}(\{0\})$. We show that $\bar{a}_{1}=\left(a_{1}^{*}, a_{1}^{*}\right)$. Suppose that $\bar{a}_{1}^{1}>\bar{a}_{1}^{2}$. Since $\frac{\partial h}{\partial a_{1}^{2}} \neq 0$ by (4), the implicit function theorem shows that there exists a function $\gamma$ defined in a neighborhood of $\bar{a}_{1}^{1}$ such that $h\left(a_{1}^{1}, \gamma\left(a_{1}^{1}\right)\right)=0$. Furthermore, $\gamma$ is differentiable at $\bar{a}_{1}^{1}$ and the derivative $\gamma^{\prime}\left(\bar{a}_{1}^{1}\right)$ is given by the left-hand side of (4) with $\bar{a}_{1}^{i}$ replacing $a_{1}^{i}$. Now let $\delta\left(a_{1}^{1}\right)=V\left(a_{1}^{1}, \gamma\left(a_{1}^{1}\right), a_{2}\right) . \delta$ is also differentiable at $\bar{a}_{1}^{1}$ and its derivative is given by

$$
\delta^{\prime}\left(\bar{a}_{1}^{1}\right)=\frac{\partial V}{\partial a_{1}^{1}}\left(\bar{a}_{1}, a_{2}\right)+\frac{\partial V}{\partial a_{1}^{2}}\left(\bar{a}_{1}, a_{2}\right) \gamma^{\prime}\left(\bar{a}_{1}^{1}\right) .
$$

It can be readily verified that $(5)$ implies $\delta^{\prime}\left(a_{1}^{1}\right)>0$. This contradicts our assumption that $V$ is maximized at $\bar{a}_{1}$ in $h^{-1}(\{0\})=0$. The symmetric argument shows that it cannot be maximized at $\bar{a}$ such that $\bar{a}_{1}^{1}>\bar{a}_{1}^{2}$ either. Hence, we must have $\bar{a}_{1}^{1}=\bar{a}_{1}^{2}=a_{1}^{*}$. //

Proof of Theorem 8: By Assumption 4, $\left(\phi_{1} * \phi_{2}\right)\left(a_{1}^{1}-a_{1}^{2}\right) \leq\left(\phi_{1} * \phi_{2}\right)(0)$. It then follows from (3) that $E^{\sigma}\left[\alpha_{2}\left(\sigma_{1}, \tilde{y}\right)\right] \leq a_{2}^{*}$. By Assumption 3, we have

$$
\begin{aligned}
v(\sigma, f) & =E^{\sigma}\left[V\left(\sigma_{1}, a_{2}^{1}=a_{2}^{2}=\alpha_{2}\left(\sigma_{1}, \tilde{y}\right)\right)\right] \\
& =A\left(\sigma_{1}\right) E^{\sigma}\left[\alpha_{2}\left(\sigma_{1}, \tilde{y}\right)\right]+B\left(\sigma_{1}\right) \\
& \leq A\left(\sigma_{1}\right) a_{2}^{*}+B\left(\sigma_{1}\right) \\
& =V\left(\sigma_{1}, a_{2}^{1}=a_{2}^{2}=a_{2}^{*}\right) .
\end{aligned}
$$


Since $\sigma_{1}$ solves (2) by assumption, we have by Lemma 7

$$
V\left(\sigma_{1}, a_{2}^{*}, a_{2}^{*}\right) \leq V\left(a_{1}^{*}, a_{1}^{*}, a_{2}^{*}, a_{2}^{*}\right) .
$$

Since the right-hand side equals the principal's expected payoff in the symmetric PBE under the no-feedback policy, the desired conclusion follows. //

Proof of Theorem 9: We first show that Assumption 5 implies

$$
\left.\left.P\left(\left|\tilde{\zeta}_{2}\right| \geq \kappa\right)\right)=\min _{\delta \in \mathbf{R}} P\left(\left|\tilde{\zeta}_{2}+\delta\right| \geq \kappa\right)\right) \text { for any } \kappa>0 .
$$

Let $\delta>0$ and $\kappa>0$ be given. When $\delta<2 \kappa$, we have

$$
\begin{aligned}
& \left.\left.P\left(\left|\tilde{\zeta}_{2}\right|<\kappa\right)\right)-P\left(\left|\tilde{\zeta}_{2}+\delta\right|<\kappa\right)\right) \\
& =-\int_{-\kappa-\delta}^{-\kappa} \phi_{2}(x) d x+\int_{\kappa-\delta}^{\kappa} \phi_{2}(x) d x \\
& >-\delta \phi_{2}(-\kappa)+\delta \phi_{2}(\kappa) \\
& =0 .
\end{aligned}
$$

On the other hand, when $\delta>2 \kappa$, we have

$$
\begin{aligned}
& \left.\left.P\left(\left|\tilde{\zeta}_{2}\right|<\kappa\right)\right)-P\left(\left|\tilde{\zeta}_{2}+\delta\right|<\kappa\right)\right) \\
& =\int_{-\kappa}^{\kappa} \phi_{2}(x) d x-\int_{-\kappa-\delta}^{\kappa-\delta} \phi_{2}(x) d x \\
& >2 \kappa \phi_{2}(\kappa)-2 \kappa \phi_{2}(\kappa-\delta) \\
& >0 .
\end{aligned}
$$

The similar argument proves (a9) when $\delta<0$.

We now show that the expected stage 2 effort implied by $\sigma$ is less than or equal to that implied by the symmetric PBE under the full-feedback policy:

$$
E^{\sigma}\left[\alpha_{2}\left(\sigma_{1}, \tilde{y}\right)\right] \leq a_{2}^{* *} \equiv \int_{\mathbf{R}}\left(c_{2}^{\prime}\right)^{-1}\left(\phi_{2}\left(x_{1}\right)\right) \phi\left(x_{1}\right) d x_{1} .
$$

It would then follow from Lemma 7 and Assumption 2 that

$$
\begin{aligned}
& E^{\sigma}\left[V\left(\sigma_{1}, a_{2}^{1}=a_{2}^{2}=\alpha_{2}\left(\sigma_{1}, \tilde{y}\right)\right)\right] \\
& \leq E^{\sigma}\left[V\left(a_{1}^{*}, a_{1}^{*}, a_{2}^{1}=a_{2}^{2}=\alpha_{2}\left(\sigma_{1}, \tilde{y}\right)\right)\right] \\
& =A\left(a_{1}^{*}, a_{1}^{*}\right) E^{\sigma}\left[\alpha_{2}\left(\sigma_{1}, \tilde{y}\right)\right]+B\left(a_{1}^{*}, a_{1}^{*}\right) \\
& \leq A\left(a_{1}^{*}, a_{1}^{*}\right) a_{2}^{* *}+B\left(a_{1}^{*}, a_{1}^{*}\right) .
\end{aligned}
$$


Since the far right-hand side equals the principal's expected payoff in the symmetric PBE under the full-feedback policy, the desired conclusion would follow.

Note that since $E^{\sigma}\left[\alpha_{2}\left(\sigma_{1}, \tilde{y}\right)\right] \leq E^{\sigma}\left[\left(c_{2}^{\prime}\right)^{-1}\left(\phi_{2}\left(\tilde{x}_{1}\right)\right)\right]$ from the proof of Theorem 5, (a10) is implied by

$$
E^{\sigma}\left[\left(c_{2}^{\prime}\right)^{-1}\left(\phi_{2}\left(\tilde{x}_{1}\right)\right)\right] \leq a_{2}^{* *}
$$

Let $\eta_{2}:\left[0, \phi_{2}(0)\right] \rightarrow \mathbf{R}_{+}$be the inverse of the restriction of $\phi_{2}$ to $\mathbf{R}_{+}$. In other words, for each $u \in\left[0, \phi_{2}(0)\right], \eta_{2}(u) \geq 0$ is the unique number such that $\phi_{2}\left(\eta_{2}(u)\right)=u$. Note that $\eta_{2}$ is well-defined under Assumption 5. Given any $\delta \in \mathbf{R}$, let the function $G(\cdot \mid \delta):\left[0, \phi_{2}(0)\right] \rightarrow$ $\mathbf{R}_{+}$be defined by $G(u \mid \delta)=1-\Phi_{2}\left(\eta_{2}(u)-\delta\right)+\Phi_{2}\left(-\eta_{2}(u)-\delta\right)=P\left(\left|\zeta_{2}+\delta\right| \geq \eta_{2}(u)\right)$. It is easy to verify that $G(\cdot \mid \delta)$ is a distribution function over $\left[0, \phi_{2}(0)\right]$ since it is increasing, and satisfies $G(0 \mid \delta)=0$ and $G\left(\phi_{2}(0) \mid \delta\right)=1$. If we write $\delta=a_{1}^{1}-a_{1}^{2}$ and $u=\phi_{2}\left(x_{1}\right)$, then

$$
\begin{aligned}
& E\left[\left(c_{2}^{\prime}\right)^{-1}\left(\phi_{2}\left(x_{1}\right)\right) \mid a_{1}\right] \\
& =\int_{\mathbf{R}}\left(c_{2}^{\prime}\right)^{-1}\left(\phi_{2}\left(x_{1}\right)\right) \phi_{1}\left(x_{1}-\delta\right) d x_{1} \\
& =\int_{0}^{\phi_{2}(0)}\left(c_{2}^{\prime}\right)^{-1}(u) \phi_{1}\left(\eta_{2}(u)-\delta\right)\left(-\eta_{2}^{\prime}(u)\right) d u \\
& +\int_{0}^{\phi_{2}(0)}\left(c_{2}^{\prime}\right)^{-1}(u) \phi_{1}\left(-\eta_{2}(u)-\delta\right)\left(-\eta_{2}^{\prime}(u)\right) d u \\
& =\int_{0}^{\phi_{2}(0)}\left(c_{2}^{\prime}\right)^{-1}(u) d G(u \mid \delta) .
\end{aligned}
$$

By (a9), $G(u \mid \delta)=P\left(\left|\zeta_{2}+\delta\right| \geq \eta_{2}(u)\right) \geq P\left(\left|\zeta_{2}\right| \geq \eta_{2}(u)\right)=G(u \mid 0)$ for any $u \in\left[0, \phi_{2}(0)\right]$ and $\delta \in \mathbf{R}$ so that $G(u \mid 0)$ first-order stochastically dominates $G(u \mid \delta)$ with $\delta \neq 0$. Since $\left(c_{2}^{\prime}\right)^{-1}$ is increasing, it follows that

$$
\int_{0}^{\phi_{2}(0)}\left(c_{2}^{\prime}\right)^{-1}(u) d G(u \mid \delta) \leq \int_{0}^{\phi_{2}(0)}\left(c_{2}^{\prime}\right)^{-1}(u) d G(u \mid 0) .
$$

Changing variables back to $x_{1}$, we see that the right-hand side of this inequality equals $a_{2}^{* *} \cdot / /$

Proof of Theorem 10: The proof consists of three steps. In the first step, we specify the effort level contingent on each possible history. The second step shows that this effort 
level must be chosen in any symmetric pure PBE. In the third step, it is shown that this effort level is indeed optimal. Define

$$
\kappa=\min \left\{1, \frac{1}{7} \min _{1 \leq t \leq T} \inf _{a_{t} \in \mathbf{R}} c_{t}^{\prime \prime}\left(a_{t}\right)\right\}>0,
$$

and take $\epsilon>0$ such that

$$
\begin{aligned}
& \max \left\{c_{t}\left(\left(c_{t}^{\prime}\right)^{-1}(\epsilon T)\right), c_{t}\left(\left(c_{t}^{\prime}\right)^{-1}(-\epsilon T)\right)\right\}<1 \text { for } t=1, \ldots, T, \\
& \epsilon<2^{-T} \kappa, \text { and } \\
& \epsilon<\frac{1}{T} \min _{1 \leq t \leq T} \min \left\{\lim _{a \rightarrow \infty} c_{t}^{\prime}(a), \lim _{a \rightarrow \infty}\left|c_{t}^{\prime}(-a)\right|\right\} .
\end{aligned}
$$

Step 1. For each $z_{t-1} \in Z_{t-1}(f)$ and $t=1, \ldots, T$, define

$$
\alpha_{t}\left(z_{t-1}\right)=\left(c_{t}^{\prime}\right)^{-1}\left(\int_{\mathbf{R}^{T-1}} \phi_{T}\left(\Delta_{T-1}\right) \prod_{s=t}^{T-1} \phi_{s}\left(x_{s}\right) g_{t-1}\left(\omega_{t-1} \mid z_{t-1}\right) d \omega_{T-1}\right) .
$$

Let $g_{t-1}^{\alpha}\left(\omega_{t-1} \mid b_{t-1}^{1}, z_{t-1}\right)$ denote the density of $\omega_{t-1}$ conditional on $z_{t-1}=\left(y_{1}, \ldots, y_{t-1}\right)$ and $b_{t-1}^{1}=\left(a_{1}^{1}, \ldots, a_{t-1}^{1}\right)$, provided that agent 2 chooses $\alpha_{s}\left(z_{s-1}\right)$ in stage $s=1, \ldots, t-1$ :

$$
g_{t-1}^{\alpha}\left(\omega_{t-1} \mid b_{t-1}^{1}, z_{t-1}\right)=\frac{\prod_{s=1}^{t-1} \phi_{s}\left(x_{s}-a_{s}^{1}+\alpha_{s}\left(z_{s-1}\right)\right)}{\int_{X_{t-1}^{f}\left(z_{t-1}\right)} \prod_{s=1}^{t-1} \phi_{s}\left(x_{s}^{\prime}-a_{s}^{1}+\alpha_{s}\left(z_{s-1}\right)\right) d \omega_{t-1}^{\prime}}
$$

if $\omega_{t-1} \in X_{t-1}^{f}\left(z_{t-1}\right)$ and $g_{t-1}^{\alpha}\left(\omega_{t-1} \mid b_{t-1}^{1}, z_{t-1}\right)=0$ otherwise. Note that for any $\omega_{t-1} \in$ $X_{t-1}^{f}\left(z_{t-1}\right)$ and $u=1, \ldots, t-1$,

$$
\begin{aligned}
& \frac{\partial g_{t-1}^{\alpha}\left(\omega_{t-1} \mid b_{t-1}^{1}, z_{t-1}\right)}{\partial a_{u}^{1}} \\
& =-\frac{\phi_{u}^{\prime}\left(x_{u}-a_{u}^{1}+\alpha_{u}\left(z_{u-1}\right)\right)}{\phi_{u}\left(x_{u}-a_{u}^{1}+\alpha_{u}\left(z_{u-1}\right)\right)} \frac{\prod_{s=1}^{t-1} \phi_{s}\left(x_{s}-a_{s}^{1}+\alpha_{s}\left(z_{s-1}\right)\right)}{\int_{X_{t-1}^{f}\left(z_{t-1}\right)} \prod_{s=1}^{t-1} \phi_{s}\left(x_{s}^{\prime}-a_{s}^{1}+\alpha_{s}\left(z_{s-1}\right)\right) d \omega_{t-1}^{\prime}} \\
& +\frac{\prod_{s=1}^{t-1} \phi_{s}\left(x_{s}-a_{s}^{1}+\alpha_{s}\left(z_{s-1}\right)\right)}{\left(\int_{X_{t-1}^{f}\left(z_{t-1}\right)} \prod_{s=1}^{t-1} \phi_{s}\left(x_{s}^{\prime}-a_{s}^{1}+\alpha_{s}\left(z_{s-1}\right)\right) d \omega_{t-1}^{\prime}\right)^{2}} \\
& \times \int_{X_{t-1}^{f}\left(z_{t-1}\right)} \frac{\phi_{u}^{\prime}\left(x_{u}^{\prime}-a_{u}^{1}+\alpha_{u}\left(z_{u-1}\right)\right)}{\phi_{u}\left(x_{u}^{\prime}-a_{u}^{1}+\alpha_{u}\left(z_{u-1}\right)\right)} \prod_{s=1}^{t-1} \phi_{s}\left(x_{s}^{\prime}-a_{s}^{1}+\alpha_{s}\left(z_{s-1}\right)\right) d \omega_{t-1}^{\prime} .
\end{aligned}
$$

Hence, when $(* *)$ holds,

$$
\begin{aligned}
& \int_{X_{t-1}^{f}\left(z_{t-1}\right)}\left|\frac{\partial g_{t-1}^{\alpha}}{\partial a_{u}^{1}}\left(\omega_{t-1} \mid b_{t-1}^{1}, z_{t-1}\right)\right| d \omega_{t-1} \\
& \leq 2 \frac{\int_{X_{t-1}^{f}\left(z_{t-1}\right)}\left|\frac{\phi_{u}^{\prime}\left(x_{u}-a_{u}^{1}+\alpha_{u}\left(z_{u-1}\right)\right)}{\phi_{u}\left(x_{u}-a_{u}^{1}+\alpha_{u}\left(z_{u-1}\right)\right)}\right| \prod_{s=1}^{t-1} \phi_{s}\left(x_{s}-a_{s}^{1}+\alpha_{s}\left(z_{s-1}\right)\right) d \omega_{t-1}}{\int_{X_{t-1}^{f}\left(z_{t-1}\right)} \prod_{s=1}^{t-1} \phi_{s}\left(x_{s}-a_{s}^{1}+\alpha_{s}\left(z_{s-1}\right)\right) d \omega_{t-1}} \\
& \leq 2 \epsilon .
\end{aligned}
$$


Now for each $b_{T-1}^{1}=\left(a_{1}^{1}, \ldots, a_{T-1}^{1}\right), z_{T-1} \in Z_{T-1}(f)$, and $a_{T}^{1} \in \mathbf{R}$, let

$$
\begin{aligned}
\varphi_{T}^{1}\left(a_{T}^{1} \mid b_{T-1}^{1}, z_{T-1}\right)=-c_{T}^{\prime}\left(a_{T}^{1}\right)+ & \int_{\mathbf{R}^{T-1}} \phi_{T}\left(a_{T}^{1}-\alpha_{T}\left(z_{T-1}\right)+\Delta_{T-1}\right) \\
& \times g_{T-1}^{\alpha}\left(\omega_{T-1} \mid b_{T-1}^{1}, z_{T-1}\right) d \omega_{T-1} .
\end{aligned}
$$

Note that $\varphi_{T}^{1}$ is continuous in $a_{T}^{1}$, and that

$$
\varphi_{T}^{1}\left(-a_{T}^{1} \mid b_{T-1}^{1}, z_{T-1}\right)>0, \text { and } \varphi_{T}^{1}\left(a_{T}^{1} \mid b_{T-1}^{1}, z_{T-1}\right)<0 \text { for } a_{T}^{1} \text { large enough }
$$

by (a12). Furthermore,

$$
\begin{aligned}
& \frac{\partial \varphi_{T}^{1}}{\partial a_{T}^{1}}\left(a_{T}^{1} \mid b_{T-1}^{1}, z_{T-1}\right) \\
& =-c_{T}^{\prime \prime}\left(a_{T}^{1}\right)+\int_{\mathbf{R}^{T-1}} \phi_{T}^{\prime}\left(a_{T}^{1}-\alpha_{T}\left(z_{T-1}\right)+\Delta_{T-1}\right) \\
& \times g_{T-1}^{\alpha}\left(\omega_{T-1} \mid b_{T-1}^{1}, z_{T-1}\right) d \omega_{T-1} \\
& \leq-\kappa+\epsilon<0
\end{aligned}
$$

for any $a_{T}^{1} \in \mathbf{R}$. Hence, there exists a unique $a_{T}^{1} \in \mathbf{R}$ that solves $\varphi_{T}^{1}\left(a_{T}^{1} \mid b_{T-1}^{1}, z_{T-1}\right)=0$. We define $\sigma_{T}^{1}\left(b_{T-1}^{1}, z_{T-1}\right)$ to be this solution. Agent 2's contingent action $\sigma_{T}^{2}$ in stage $T$ is defined in a similar manner: For each $b_{T-1}^{2}=\left(a_{1}^{2}, \ldots, a_{T-1}^{2}\right), z_{T-1} \in Z_{T-1}(f)$, and $a_{T}^{2} \in \mathbf{R}$, let $\sigma_{T}^{2}\left(b_{T-1}^{2}, z_{T-1}\right)$ be the unique solution to $\varphi_{T}^{2}\left(a_{T}^{2} \mid b_{T-1}^{2}, z_{T-1}\right)=0$, where

$$
\begin{aligned}
\varphi_{T}^{2}\left(a_{T}^{2} \mid b_{T-1}^{2}, z_{T-1}\right)=-c_{T}^{\prime}\left(a_{T}^{2}\right)+\int_{\mathbf{R}^{T-1}} & \phi_{T}\left(-\alpha_{T}\left(z_{T-1}\right)+a_{T}^{2}-\Delta_{T-1}\right) \\
& \times g_{T-1}^{\alpha}\left(\omega_{T-1} \mid b_{T-1}^{2}, z_{T-1}\right) d \omega_{T-1} .
\end{aligned}
$$

We now show that $\sigma_{T}^{i}$ defined above satisfies (i) and (ii) below.

(i) $c_{T}\left(\sigma_{T}^{i}\left(b_{T-1}^{i}, z_{T-1}\right)\right)<1$ and $\left|c_{T}^{\prime}\left(\sigma_{T}^{i}\left(b_{T-1}^{i}, z_{T-1}\right)\right)\right|<1$ for any $\left(b_{T-1}^{i}, z_{T-1}\right)$.

(ii) For $u=1, \ldots, T-1, \sigma_{T}^{i}$ is differentiable as a function of $a_{u}^{1}$, and

$$
\left|\frac{\partial \sigma_{T}^{i}}{\partial a_{u}^{1}}\left(b_{T-1}^{i}, z_{T-1}\right)\right|<1 \text { for any }\left(b_{T-1}^{i}, z_{T-1}\right)
$$

It is clear from the definition of $\sigma_{T}^{i}$ and $(* *)$ that $\left|c_{T}^{\prime}\left(\sigma_{T}^{i}\right)\right| \leq \epsilon<1$ and hence that

$$
c_{T}\left(\sigma_{T}^{i}\right) \leq \max \left\{c_{T}\left(\left(c_{T}^{\prime}\right)^{-1}(\epsilon)\right), c_{T}\left(\left(c_{T}^{\prime}\right)^{-1}(-\epsilon)\right)\right\}<1
$$


Since $\frac{\partial \varphi_{T}^{1}}{\partial a_{T}^{1}}\left(a_{T}^{1} \mid b_{T-1}^{1}, z_{T-1}\right)>0$ as noted above, $\sigma_{T}^{1}$ is differentiable with respect to $a_{u}^{1}$ by the implicit function theorem, and the derivative is given by

$$
\frac{\partial \sigma_{T}^{1}}{\partial a_{u}^{1}}\left(b_{T-1}^{1}, z_{T-1}\right)=-\frac{\frac{\partial \varphi_{T}^{1}}{\partial a_{u}^{1}}\left(\sigma_{T}^{1}\left(b_{T-1}^{1}, z_{T-1}\right) \mid b_{T-1}^{1}, z_{T-1}\right)}{\frac{\partial \varphi_{T}^{1}}{\partial a_{T}^{1}}\left(\sigma_{T}^{1}\left(b_{T-1}^{1}, z_{T-1}\right) \mid b_{T-1}^{1}, z_{T-1}\right)}
$$

Since

$$
\frac{\partial \varphi_{T}^{1}}{\partial a_{u}^{1}}\left(b_{T-1}^{1}, z_{T-1}\right)=\int_{\mathbf{R}^{T-1}} \phi_{T}\left(\sigma_{T}^{1}-\alpha_{T}+\Delta_{T-1}\right) \frac{\partial g_{T-1}^{\alpha}}{\partial a_{u}^{1}}\left(\omega_{T-1} \mid b_{T-1}^{1}, z_{T-1}\right) d \omega_{T-1}
$$

we have $\left|\frac{\partial \varphi_{T}^{1}}{\partial a_{u}^{1}}\right| \leq 2 \epsilon^{2}$ by (a13). Hence, it follows from (a14) that

$$
\left|\frac{\partial \sigma_{T}^{1}}{\partial a_{u}^{1}}\left(b_{T-1}^{1}, z_{T-1}\right)\right| \leq \frac{2 \epsilon^{2}}{\kappa-\epsilon}<1
$$

As an induction hypothesis, fix $t<T$ and suppose that we have defined $\sigma_{T}^{i}, \ldots, \sigma_{t+1}^{i}$ for which (i) and (ii) below hold $(s=t+1, \ldots, T)$ :

(i) $c_{s}\left(\sigma_{s}^{i}\left(b_{s-1}^{i}, z_{s-1}\right)\right)<1$ and $\left|c_{s}^{\prime}\left(\sigma_{s}^{i}\left(b_{s-1}^{i}, z_{s-1}\right)\right)\right|<1$ for any $\left(b_{s-1}^{i}, z_{s-1}\right)$.

(ii) For $u=1, \ldots, s-1, \sigma_{s}^{i}\left(b_{s-1}^{i}, z_{s-1}\right)$ is differentiable as a function of $a_{u}^{1}$, and

$$
\left|\frac{\partial \sigma_{s}^{i}}{\partial a_{u}^{1}}\left(b_{s-1}^{i}, z_{s-1}\right)\right|<1 \quad \text { for any }\left(b_{s-1}^{i}, z_{s-1}\right)
$$

Let

$$
\sigma_{t+1, t}^{i}\left(b_{t}^{i}, z_{t}\right)=\sigma_{t+1}^{i}\left(b_{t}^{i}, z_{t}\right)
$$

and for each $s=t+2, \ldots, T$, define $\sigma_{s, t}^{i}$ recursively by

$$
\sigma_{s, t}^{i}\left(b_{t}^{i}, z_{s-1}\right)=\sigma_{s}^{i}\left(\left(b_{t}^{i}, \sigma_{t+1, t}^{i}\left(b_{t}^{i}, z_{t}\right), \ldots, \sigma_{s-1, t}^{i}\left(b_{t}^{i}, z_{s-2}\right)\right), z_{s-1}\right)
$$

The interpretation is that $\sigma_{s, t}^{i}\left(b_{t}^{i}, z_{s-1}\right)$ is agent $i$ 's action in stage $s$ induced by $\sigma_{t+1}^{i}, \ldots, \sigma_{s}^{i}$ after the sequence of actions $b_{t}^{i}$ in stages $1, \ldots, t$ and announcements $z_{s-1}$ after stages $1, \ldots, s-1$. It can be verified that for any $u \leq t<s$,

$$
\frac{\partial \sigma_{s, t}^{i}}{\partial a_{u}^{i}}=\sum_{k=0}^{s-t-1} \sum_{t<\tau_{1}<\cdots<\tau_{k}<s} \frac{\partial \sigma_{s}^{i}}{\partial a_{\tau_{k}}^{i}} \frac{\partial \sigma_{\tau_{k}}^{i}}{\partial a_{\tau_{k-1}}^{i}} \cdots \frac{\partial \sigma_{\tau_{2}}^{i}}{\partial a_{\tau_{1}}^{i}} \frac{\partial \sigma_{\tau_{1}}^{i}}{\partial a_{u}^{i}}
$$


We now define $\sigma_{t}^{1}$ as follows. For $b_{t-1}^{1}=\left(a_{1}^{1}, \ldots, a_{t-1}^{1}\right), z_{t-1} \in Z_{t-1}(f)$, and $a_{t}^{1} \in \mathbf{R}$, let

$$
\begin{aligned}
& \varphi_{t}^{1}\left(a_{t}^{1} \mid b_{t-1}, z_{t-1}\right)=-c_{t}^{\prime}\left(a_{t}^{1}\right) \\
& +\int_{\mathbf{R}^{T-1}}\left\{\Phi_{T}\left(\sigma_{T, t}^{1}\left(\left(b_{t-1}^{1}, a_{t}^{1}\right), z_{T-1}\right)-\alpha_{T}\left(z_{T-1}\right)+\Delta_{T-1}\right)\right. \\
& \left.\quad-\sum_{s=t+1}^{T} c_{s}\left(\sigma_{s, t}^{1}\left(\left(b_{t-1}^{1}, a_{t}^{1}\right), z_{s-1}\right)\right)\right\} \\
& \quad \times \phi_{t}^{\prime}\left(x_{t}-a_{t}^{1}+\alpha_{t}\left(z_{t-1}\right)\right) \prod_{s=t+1}^{T-1} \phi_{s}\left(x_{s}-\sigma_{s, t}^{1}\left(\left(b_{t-1}^{1}, a_{t}^{1}\right), z_{s-1}\right)+\alpha_{s}\left(z_{s-1}\right)\right) \\
& \quad \times g_{t-1}^{\alpha}\left(\omega_{t-1} \mid b_{t-1}^{1}, z_{t-1}\right) d \omega_{T-1},
\end{aligned}
$$

where $z_{s-1}=\left(z_{t-1}, f_{t}\left(\omega_{t}\right), \ldots, f_{s-1}\left(w_{s-1}\right)\right)$ for $s=t+1, \ldots, T$. By the induction hypothesis, $\varphi_{t}^{1}$ is differentiable in $a_{t}^{1}$, and the derivative is given by

$$
\begin{aligned}
& \frac{\partial \varphi_{t}^{1}}{\partial a_{t}^{1}}\left(a_{t}^{1} \mid b_{t-1}^{1}, z_{t-1}\right) \\
& =-c_{t}^{\prime \prime}\left(a_{t}^{1}\right)+\int_{\mathbf{R}^{T-1}}\left\{\phi_{T}\left(\sigma_{T, t}^{1}-\alpha_{T}+\Delta_{T-1}\right) \frac{\partial \sigma_{T, t}^{1}}{\partial a_{t}^{1}}-\sum_{s=t+1}^{T} c_{s}^{\prime}\left(\sigma_{s, t}^{1}\right) \frac{\partial \sigma_{s, t}^{1}}{\partial a_{t}^{1}}\right\} \\
& \times \frac{\phi_{t}^{\prime}\left(x_{t}-a_{t}^{1}+\sigma_{t}^{2}\right)}{\phi_{t}\left(x_{t}-a_{t}^{1}+\alpha_{t}\right)} \prod_{s=t}^{T-1} \phi_{s}\left(x_{s}-\sigma_{s, t}^{1}+\alpha_{s}\right) g_{t-1}^{\alpha}\left(\omega_{t-1} \mid b_{t-1}^{1}, z_{t-1}\right) d \omega_{T-1} \\
& -\int_{\mathbf{R}^{T-1}}\left\{\Phi_{T}\left(\sigma_{T, t}^{1}-\alpha_{T}+\Delta_{T-1}\right)-\sum_{s=t+1}^{T} c_{s}\left(\sigma_{s, t}^{1}\right)\right\} \\
& \times\left\{\phi_{t}^{\prime \prime}\left(x_{t}-a_{t}^{1}+\alpha_{t}\right)+\phi_{t}^{\prime}\left(x_{t}-a_{t}^{1}+\alpha_{t}\right) \sum_{k=t+1}^{T-1} \frac{\phi_{k}^{\prime}\left(x_{k}-\sigma_{k, t}^{1}+\alpha_{k}\right)}{\phi_{k}\left(x_{k}-\sigma_{k, t}^{1}+\alpha_{k}\right)} \frac{\partial \sigma_{k, t}}{\partial a_{t}^{1}}\right\} \\
& \times \prod_{s=t+1}^{T-1} \phi_{s}\left(x_{s}-\sigma_{s, t}^{1}+\alpha_{s}\right) g_{t-1}^{\alpha}\left(\omega_{t-1} \mid b_{t-1}^{1}, z_{t-1}\right) d \omega_{T-1} .
\end{aligned}
$$

Note first that by the induction hypothesis,

$$
\begin{aligned}
\left|\frac{\partial \sigma_{s, t}^{i}}{\partial a_{t}^{i}}\right| & =\sum_{k=0}^{s-t-1} \sum_{t<\tau_{1}<\cdots<\tau_{k}<s}\left|\frac{\partial \sigma_{s}^{i}}{\partial a_{\tau_{k}}^{i}}\right|\left|\frac{\partial \sigma_{\tau_{k}}^{i}}{\partial a_{\tau_{k-1}}^{i}}\right| \cdots\left|\frac{\partial \sigma_{\tau_{2}}^{i}}{\partial a_{\tau_{1}}^{i}}\right|\left|\frac{\partial \sigma_{\tau_{1}}^{i}}{\partial a_{t}^{i}}\right| \\
& <\sum_{k=0}^{s-t-1} \sum_{t<\tau_{1}<\cdots<\tau_{k}<s} 1 \\
& =\sum_{k=0}^{s-t-1}\left(\begin{array}{c}
s-t-1 \\
k
\end{array}\right) \\
& =2^{s-t-1}
\end{aligned}
$$


Hence, we can evaluate $\frac{\partial \varphi_{t}^{1}}{\partial a_{t}^{1}}$ using (a12) as:

$$
\begin{aligned}
& \frac{\partial \varphi_{t}^{1}}{\partial a_{t}^{1}}\left(a_{t}^{1} \mid b_{t-1}^{1}, z_{t-1}\right) \\
& \leq-c_{t}^{\prime \prime}\left(a_{t}^{1}\right)+\epsilon\left\{\epsilon 2^{T-t-1}+\sum_{s=t+1}^{T} 2^{s-t-1}\right\}+\epsilon\{1+T-t\}\left\{1+\epsilon \sum_{s=t+1}^{T-1} 2^{k-t-1}\right\} \\
& =-c_{t}^{\prime \prime}\left(a_{t}^{1}\right)+\epsilon\left(\epsilon 2^{T-t-1}+2^{T-t}-1\right)+\epsilon(1+T-t)\left(1+\epsilon 2^{T-t-1}-\epsilon\right) \\
& \leq-c_{t}^{\prime \prime}\left(a_{t}^{1}\right)+\epsilon\left(\kappa+2^{T-t}-1\right)+\kappa(1+\kappa) \\
& \leq-c_{t}^{\prime \prime}\left(a_{t}^{1}\right)+3 \kappa<0 .
\end{aligned}
$$

This, along with the fact that

$$
\varphi_{t}^{1}\left(-a_{t}^{1} \mid b_{t-1}^{1}, z_{t-1}\right)>0 \text { and } \varphi_{t}^{1}\left(a_{t}^{1} \mid b_{t-1}^{1}, z_{t-1}\right)<0 \text { for } a_{t}^{1} \text { large enough, }
$$

implies that there exists a unique $a_{t}^{1}$ for which $\varphi_{t}^{1}\left(a_{t}^{1} \mid b_{t-1}^{1}, z_{t-1}\right)=0$. Define $\sigma_{t}^{1}\left(b_{t-1}^{1}, z_{t-1}\right)$ to be this solution.

For agent 2 , for each $b_{t-1}^{2}=\left(a_{1}^{2}, \ldots, a_{t-1}^{2}\right)$, and $z_{t-1} \in Z_{t-1}(f)$, let $\sigma_{t}^{2}\left(b_{t-1}^{2}, z_{t-1}\right)$ be the unique solution to $\varphi_{t}^{2}\left(a_{t}^{2} \mid b_{t-1}^{2}, z_{t-1}\right)=0$, where

$$
\begin{aligned}
& \varphi_{t}^{2}\left(a_{t}^{2} \mid b_{t-1}^{2}, z_{t-1}\right)=-c_{t}^{\prime}\left(a_{t}^{2}\right) \\
& +\int_{\mathbf{R}^{T-1}}\left\{-\Phi_{T}\left(\alpha_{T}\left(z_{T-1}\right)+a_{t}^{2}-\Delta_{T-1}\right)-\sum_{s=t+1}^{T} c_{s}\left(\sigma_{s, t}^{2}\left(\left(b_{t-1}^{2}, a_{t}^{2}\right), z_{s-1}\right)\right)\right\} \\
& \quad \times \phi_{t}^{\prime}\left(x_{t}-\alpha_{t}\left(z_{t-1}\right)+a_{t}^{2}\right) \prod_{s=t+1}^{T-1} \phi_{s}\left(x_{s}-\alpha_{s}\left(z_{s-1}\right)+\sigma_{s, t}^{2}\left(\left(b_{t-1}^{2}, a_{t}^{2}\right), z_{s-1}\right)\right) \\
& \quad \times g_{t-1}^{\alpha}\left(\omega_{t-1} \mid b_{t-1}^{2}, z_{t-1}\right) d \omega_{T-1} .
\end{aligned}
$$

To see that $\sigma_{t}^{1}$ satisfies (i), note that (a17) and (**) together imply

$$
\left|c_{t}^{\prime}\left(\sigma_{t}^{1}\left(b_{t-1}^{1}, z_{t-1}\right)\right)\right| \leq \epsilon(1+T-t) \leq \epsilon T<1
$$

This further implies that $\left(c_{t}^{\prime}\right)^{-1}(-\epsilon T)<\sigma_{t}^{1}\left(b_{t-1}^{1}, z_{t-1}\right)<\left(c_{t}^{\prime}\right)^{-1}(\epsilon T)$. Hence,

$$
c_{t}\left(\sigma_{t}^{1}\left(b_{t-1}^{1}, z_{t-1}\right)\right)<\max \left\{c_{t}\left(\left(c_{t}^{\prime}\right)^{-1}(-\epsilon T)\right), c_{t}\left(\left(c_{t}^{\prime}\right)^{-1}(\epsilon T)\right)\right\}<1
$$

For (ii), since $\frac{\partial \varphi_{t}^{1}}{\partial a_{t}^{1}}\left(a_{t}^{1} \mid b_{t-1}^{1}, z_{t-1}\right)<0, \sigma_{t}^{1}$ is differentiable as a function of $a_{u}^{1}(u=$ $1, \ldots, t-1)$ by the implicit function theorem. Furthermore, differentiation of $\varphi_{t}^{1}$ with 
respect to $a_{u}^{1}$ yields

$$
\begin{aligned}
& \frac{\partial \varphi_{t}^{1}}{\partial a_{u}^{1}}\left(a_{t}^{1} \mid b_{t-1}^{1}, z_{t-1}\right) \\
& =\int_{\mathbf{R}^{T-1}}\left\{\phi_{T}\left(\sigma_{T, t}^{1}-\alpha_{T}+\Delta_{T-1}\right) \frac{\partial \sigma_{T, t}^{1}}{\partial a_{u}^{1}}-\sum_{s=t+1}^{T} c_{s}^{\prime}\left(\sigma_{s, t}^{1}\right) \frac{\partial \sigma_{s, t}^{1}}{\partial a_{u}^{1}}\right\} \\
& \quad \times \phi_{t}^{\prime}\left(x_{t}-a_{t}^{1}+\alpha_{t}\right) \prod_{s=t+1}^{T-1} \phi_{s}\left(x_{s}-\sigma_{s, t}^{1}+\alpha_{s}\right) g_{t-1}^{\alpha}\left(\omega_{t-1} \mid b_{t-1}^{1}, z_{t-1}\right) d \omega_{T-1} \\
& +\int_{\mathbf{R}^{T-1}}\left\{\Phi_{T}\left(\sigma_{T, t}^{1}-\alpha_{T}+\Delta_{T-1}\right)-\sum_{s=t+1}^{T} c_{s}\left(\sigma_{s, t}^{1}\right)\right\} \\
& \times\left\{\frac{\partial g_{t-1}^{\alpha}}{\partial a_{u}^{1}}\left(\omega_{t-1} \mid b_{t-1}^{1}, z_{t-1}\right)-g_{t-1}^{\alpha}\left(\omega_{t-1} \mid b_{t-1}^{1}, z_{t-1}\right) \sum_{k=t+1}^{T-1} \frac{\phi_{k}^{\prime}\left(x_{k}-\sigma_{k, t}^{1}+\alpha_{k}\right)}{\phi_{k}\left(x_{k}-\sigma_{k, t}^{1}+\alpha_{k}\right)} \frac{\partial \sigma_{k, t}^{1}}{\partial a_{u}^{1}}\right\} \\
& \times \phi_{t}^{\prime}\left(x_{t}-a_{t}^{1}+\alpha_{t}\right) \prod_{s=t+1}^{T-1} \phi_{s}\left(x_{s}-\sigma_{s, t}^{1}+\alpha_{s}\right) d \omega_{T-1} .
\end{aligned}
$$

By $(* *),(\mathrm{a} 12),(\mathrm{a} 13),(\mathrm{a} 18)$, and the induction hypothesis, $\frac{\partial \varphi_{t}^{1}}{\partial a_{u}^{1}}$ can be evaluated as:

$$
\begin{aligned}
& \left|\frac{\partial \varphi_{t}^{1}}{\partial a_{u}^{1}}\left(a_{t}^{1} \mid b_{t-1}^{1}, z_{t-1}\right)\right| \\
& \leq\left\{\epsilon 2^{T-t-1}+\sum_{s=t+1}^{T} 2^{s-t-1}\right\} \epsilon+(1+T-t) \epsilon^{2} \sum_{k=t+1}^{T-1} 2^{k-t-1}+2 \epsilon(1+T-t) \epsilon^{T-t} \\
& =\epsilon\left(\epsilon 2^{T-t-1}+2^{T-t}-1\right)+\epsilon^{2}(1+T-t)\left(2^{T-t-1}-1\right)+2 \epsilon(1+T-t) \\
& \leq \epsilon\left(\kappa+2^{T-t}-1\right)+\kappa \epsilon 2^{T-t-1}+2 \kappa \\
& \leq \kappa+\kappa^{2}+2 \kappa \\
& \leq 4 \kappa .
\end{aligned}
$$

Therefore, the derivative $\frac{\partial \sigma_{t}^{1}}{\partial a_{u}^{1}}$ satisfies

$$
\left|\frac{\partial \sigma_{t}^{1}}{\partial a_{u}^{1}}\left(b_{t-1}^{1}, z_{t-1}\right)\right|=\frac{\left|\frac{\partial \varphi_{t}^{1}}{\partial a_{u}^{1}}\left(\sigma_{t}^{1}\left(b_{t-1}^{1}, z_{t-1}\right) \mid b_{t-1}^{1}, z_{t-1}\right)\right|}{\left|\frac{\partial \varphi_{t}^{1}}{\partial a_{t}^{1}}\left(\sigma_{t}^{1}\left(b_{t-1}^{1}, z_{t-1}\right) \mid b_{t-1}^{1}, z_{t-1}\right)\right|}<\frac{4 \kappa}{c_{t}^{\prime \prime}\left(a_{t}^{1}\right)-3 \kappa} \leq 1 .
$$

This advances the induction step and the desired conclusion follows.

Step 2. We now show that the effort choice $a_{t}^{i}=\sigma_{t}^{i}\left(b_{t-1}^{i}, z_{t-1}\right)$ in any symmetric PBE $\sigma$ must satisfy $\varphi_{t}^{i}\left(a_{t}^{i} \mid b_{t-1}^{i}, z_{t-1}\right)=0$ for any $\left(b_{t-1}^{i}, z_{t-1}\right)$ and $t$. 
Fix any symmetric PBE $\sigma$ and recall that $\pi_{t}^{i}\left(a_{t}^{i} \mid \sigma, b_{t-1}^{i}, z_{t-1}\right)$ denotes agent $i$ 's payoff over stages $t, \ldots, T$ when (i) $i$ 's history in stages $1, \ldots, t-1$ equals $h_{t-1}^{i}=\left(b_{t-1}^{i}, z_{t-1}\right)$, (ii) $i$ takes action $a_{t}^{i}$ in stage $t$ and plays according to $\sigma_{s}^{i}$ in stages $s=t+1, \ldots, T$ (given $h_{t-1}^{i}$ and $a_{t}^{i}$ ), and (iii) $j$ plays according to $\sigma_{s}^{j}$ in every stage $s$. Write $\pi_{t}^{i}\left(a_{t}^{i} \mid b_{t-1}^{i}, z_{t-1}\right)=$ $\pi_{t}^{i}\left(a_{t}^{i} \mid \sigma, b_{t-1}^{i}, z_{t-1}\right)$ for simplicity. Recall also that $g_{t-1}^{\sigma}\left(\omega_{t-1} \mid b_{t-1}^{i}, z_{t-1}\right)$ is defined as the density of $\omega_{t-1}$ conditional on $i$ 's history $\left(b_{t-1}^{i}, z_{t-1}\right)$ provided that agent $j$ played according to $\sigma_{s}^{j}$ in stage $s=1, \ldots, t-1$. For agent $1, \pi_{t}^{1}\left(a_{t}^{1} \mid b_{t-1}^{1}, z_{t-1}\right)$ can be expressed as

$$
\begin{aligned}
& \pi_{t}^{1}\left(a_{t}^{1} \mid b_{t-1}^{1}, z_{t-1}\right) \\
& =-c_{t}\left(a_{t}^{1}\right) \\
& +\int_{\mathbf{R}^{T-1}}\left\{P\left(\tilde{\zeta}_{T}+\sigma_{T, t}^{1}\left(b_{t-1}^{1}, a_{t}^{1}, z_{T-1}\right)-\sigma_{T, 0}^{2}\left(z_{T-1}\right)>-\Delta_{T-1}\right)\right. \\
& \left.\quad-\sum_{s=t+1}^{T} c_{s}\left(\sigma_{s, t}^{1}\left(b_{t-1}^{1}, a_{t}^{1}, z_{s-1}\right)\right)\right\} \\
& \times \phi_{t}\left(x_{t}-a_{t}^{1}+\sigma_{t, 0}^{2}\left(z_{t-1}\right)\right) \\
& \times \prod_{s=t+1}^{T-1} \phi_{s}\left(x_{s}-\sigma_{s, t}^{1}\left(b_{t-1}^{1}, a_{t}^{1}, z_{s-1}\right)+\sigma_{s, 0}^{2}\left(z_{s-1}\right)\right) \\
& \times g_{t-1}^{\sigma}\left(\omega_{t-1} \mid b_{t-1}^{1}, z_{t-1}\right) d \omega_{T-1},
\end{aligned}
$$

where $z_{s}=\left(z_{t-1}, f_{t}\left(\omega_{t}\right), \ldots, f_{s}\left(\omega_{s}\right)\right)$ for $s=t, \ldots, T-1$. Suppose first that $t=T$. In this case, $\pi_{T}^{1}$ can be expressed as

$$
\begin{aligned}
& \pi_{T}^{1}\left(a_{T}^{1} \mid b_{T-1}^{1}, z_{T-1}\right) \\
& =-c_{T}\left(a_{T}^{1}\right)+\int_{\mathbf{R}^{T-1}} \Phi_{T}\left(a_{T}^{1}-\sigma_{T, 0}^{2}\left(z_{T-1}\right)+\Delta_{T-1}\right) g_{T-1}^{\sigma}\left(\omega_{T-1} \mid b_{T-1}^{1}, z_{T-1}\right) d \omega_{T-1} .
\end{aligned}
$$

Differentiating $\pi_{T}^{1}$ with respect to $a_{T}^{1}$, we obtain

$$
\begin{aligned}
& \frac{\partial \pi_{T}^{1}}{\partial a_{T}^{1}}\left(a_{T}^{1} \mid b_{T-1}^{1}, z_{T-1}\right) \\
& =-c_{T}^{\prime}\left(a_{T}^{1}\right)+\int_{\mathbf{R}^{T-1}} \phi_{T}\left(a_{T}^{1}-\sigma_{T, 0}^{2}\left(z_{T-1}\right)+\Delta_{T-1}\right) g_{T-1}^{\sigma}\left(\omega_{T-1} \mid b_{T-1}^{1}, z_{T-1}\right) d \omega_{T-1} .
\end{aligned}
$$

Sequential rationality of $\sigma_{T}^{1}$ implies

$$
\begin{aligned}
c_{T}^{\prime}\left(\sigma_{T}^{1}\left(b_{T-1}^{1}, z_{T-1}\right)\right)=\int_{\mathbf{R}^{T-1}} & \phi_{T}\left(\sigma_{T}^{1}\left(b_{T-1}^{1}, z_{T-1}\right)-\sigma_{T, 0}^{2}\left(z_{T-1}\right)+\Delta_{T-1}\right) \\
& \times g_{T-1}^{\sigma}\left(\omega_{T-1} \mid b_{T-1}^{1}, z_{T-1}\right) d \omega_{T-1} .
\end{aligned}
$$


The corresponding FOC for agent 2 is given by

$$
\begin{aligned}
c_{T}^{\prime}\left(\sigma_{T}^{2}\left(b_{T-1}^{2}, z_{T-1}\right)\right)=\int_{\mathbf{R}^{T-1}} & \phi_{T}\left(-\sigma_{T, 0}^{1}\left(z_{T-1}\right)+\sigma_{T}^{2}\left(b_{T-1}^{2}, z_{T-1}\right)-\Delta_{T-1}\right) \\
& \times g_{T-1}^{\sigma}\left(\omega_{T-1} \mid b_{T-1}^{2}, z_{T-1}\right) d \omega_{T-1} .
\end{aligned}
$$

When $b_{T-1}^{i}$ equals the action sequence induced by $\sigma^{i}$ along $z_{T-1}$, we have $\sigma_{T}^{i}\left(b_{T-1}^{i}, z_{T-1}\right)=$ $\sigma_{T, 0}^{i}\left(z_{T-1}\right)$ and $g_{T-1}^{\sigma}\left(\omega_{T-1} \mid b_{T-1}^{i}, z_{T-1}\right)=g_{T-1}\left(\omega_{T-1} \mid z_{T-1}\right)$ by definition so that (a21) and (a22) imply that the stage $T$ effort on the equilibrium path should satisfy

$$
\sigma_{T, 0}^{1}\left(z_{T-1}\right)=\sigma_{T, 0}^{2}\left(z_{T-1}\right)=\left(c_{T}^{\prime}\right)^{-1}\left(E^{\sigma}\left[\phi_{T}\left(\tilde{\Delta}_{T-1}\right) \mid z_{T-1}\right]\right)=\alpha_{T}\left(z_{T-1}\right) .
$$

It follows from (a23) that (a17) and (a19) are equivalent to $\varphi_{T}^{1}\left(a_{T}^{1} \mid b_{T-1}^{1}, z_{T-1}\right)=0$ and $\varphi_{T}^{2}\left(a_{T}^{2} \mid b_{T-1}^{2}, z_{T-1}\right)=0$, respectively.

As an induction hypothesis, fix $t<T$ and suppose that the FOC for agent $i$ 's payoff maximization in stage $s$ is given by $\varphi_{s}^{i}\left(a_{s}^{i} \mid b_{s-1}^{i}, z_{s-1}\right)=0(s=t+1, \ldots, T)$. By Step $1, \sigma_{s, t}^{i}(s=t+1, \ldots, T)$ (defined in (a15) and (a16)) is differentiable as a function of $a_{t}^{1}$, and hence so is $\pi_{t}^{1}\left(\cdot \mid b_{t-1}^{1}, z_{t-1}\right)$. Using the envelope theorem, we can differentiate (a20) to obtain

$$
\begin{aligned}
& \frac{\partial \pi_{t}^{1}}{\partial a_{t}^{1}}\left(a_{t}^{1} \mid b_{t-1}^{1}, z_{t-1}\right) \\
& =-c_{t}^{\prime}\left(a_{t}^{1}\right) \\
& -\int_{\mathbf{R}^{T-1}}\left\{\Phi_{T, t}\left(\sigma_{T, t}^{1}\left(b_{t-1}^{1}, a_{t}^{1}, z_{T-1}\right)-\sigma_{T, 0}^{2}\left(z_{T-1}\right)-\Delta_{T-1}\right)\right. \\
& \left.\quad-\sum_{s=t+1}^{T} c_{s}\left(\sigma_{s, t}^{1}\left(b_{t-1}^{1}, a_{t}^{1}, z_{s-1}\right)\right)\right\} \\
& \quad \times \phi_{t}^{\prime}\left(x_{t}-a_{t}^{1}+\sigma_{t, 0}^{2}\left(z_{t-1}\right)\right) \prod_{s=t+1}^{T-1} \phi_{s}\left(x_{s}-\sigma_{s, t}^{1}\left(b_{t-1}^{1}, a_{t}^{1}, z_{s-1}\right)+\sigma_{s, 0}^{2}\left(z_{s-1}\right)\right) \\
& \quad \times g_{t-1}^{\sigma}\left(\omega_{t-1} \mid b_{t-1}^{1}, z_{t-1}\right) d \omega_{T-1} .
\end{aligned}
$$


Sequential rationality of $\sigma_{t}^{1}$ implies

$$
\begin{aligned}
c_{t}^{\prime}\left(\sigma_{t}^{1}\left(b_{t-1}^{1}, z_{t-1}\right)\right) & =-\int_{\mathbf{R}^{T-1}}\left\{\Phi_{T}\left(\sigma_{T, t-1}^{1}\left(b_{t-1}^{1}, z_{T-1}\right)-\sigma_{T, 0}^{2}\left(z_{T-1}\right)+\Delta_{T-1}\right)\right. \\
& \left.-\sum_{s=t+1}^{T} c_{s}\left(\sigma_{s, t-1}^{1}\left(b_{t-1}^{1}, z_{s-1}\right)\right)\right\} \\
& \times \phi_{t}^{\prime}\left(x_{t}-\sigma_{t}^{1}\left(b_{t-1}^{1}, z_{t-1}\right)+\sigma_{t, 0}^{2}\left(z_{t-1}\right)\right) \\
& \times \prod_{s=t+1}^{T-1} \phi_{s}\left(x_{s}-\sigma_{s, t-1}^{1}\left(b_{t-1}^{1}, z_{s-1}\right)+\sigma_{s, 0}^{2}\left(z_{s-1}\right)\right) \\
& \times g_{t-1}^{\sigma}\left(\omega_{t-1} \mid b_{t-1}^{1}, z_{t-1}\right) d \omega_{T-1} .
\end{aligned}
$$

The corresponding FOC for agent 2 is given by

$$
\begin{aligned}
c_{t}^{\prime}\left(\sigma_{t}^{1}\left(b_{t-1}^{1}, z_{t-1}\right)\right) & =-\int_{\mathbf{R}^{T-1}}\left\{\Phi_{T}\left(-\sigma_{T, 0}^{1}\left(z_{T-1}\right)+\sigma_{T, t-1}^{2}\left(b_{t-1}^{2}, z_{T-1}\right)-\Delta_{T-1}\right)\right. \\
& \left.-\sum_{s=t+1}^{T} c_{s}\left(\sigma_{s, t-1}^{2}\left(b_{t-1}^{2}, z_{s-1}\right)\right)\right\} \\
& \times \phi_{t}^{\prime}\left(x_{t}-\sigma_{t, 0}^{1}\left(z_{t-1}\right)+\sigma_{t}^{2}\left(b_{t-1}^{2}, z_{t-1}\right)\right) \\
& \times \prod_{s=t+1}^{T-1} \phi_{s}\left(x_{s}-\sigma_{s, 0}^{1}\left(z_{s-1}\right)+\sigma_{s, t-1}^{2}\left(b_{t-1}^{2}, z_{s-1}\right)\right) \\
& \times g_{t-1}^{\sigma}\left(\omega_{t-1} \mid b_{t-1}^{2}, z_{t-1}\right) d \omega_{T-1} .
\end{aligned}
$$

When $b_{t-1}^{1}$ equals the action sequence induced by $\sigma^{1}$ along $z_{t-1}, \sigma_{s, t-1}^{1}\left(b_{t-1}^{1}, z_{s-1}^{1}\right)=$ $\sigma_{s, 0}^{1}\left(z_{s-1}\right)(s=t, \ldots, T)$. Substituting this and the symmetry condition $\sigma_{s, 0}^{1}\left(z_{s-1}\right)=$ $\sigma_{s, 0}^{2}\left(z_{s-1}\right)$ for each $s$ into (a23), we obtain

$$
\begin{aligned}
c_{t}^{\prime}\left(\sigma_{t, 0}^{1}\left(z_{t-1}\right)\right) \\
=-\int_{\mathbf{R}^{T-1}} \Phi_{T}\left(\Delta_{T-1}\right) \phi_{t}^{\prime}\left(x_{t}\right) \prod_{s=t+1}^{T-1} \phi_{s}\left(x_{s}\right) g_{t-1}\left(\omega_{t-1} \mid z_{t-1}\right) d \omega_{T-1} \\
(\mathrm{a} 26) \quad+\int_{\mathbf{R}^{T-1}} \sum_{s=t}^{T} c_{s}\left(\sigma_{s, 0}^{1}\left(z_{s-1}\right)\right) \phi_{t}^{\prime}\left(x_{t}\right) \prod_{s=t+1}^{T-1} \phi_{s}\left(x_{s}\right) g_{t-1}\left(\omega_{t-1} \mid z_{t-1}\right) d \omega_{T-1} \\
=E^{\sigma}\left[\phi_{T}\left(\tilde{\Delta}_{T-1}\right) \mid z_{t-1}\right] \\
+\int_{\mathbf{R}^{T-1}} \sum_{s=t+1}^{T} c_{s}\left(\sigma_{s, 0}^{1}\left(z_{s-1}\right)\right) \phi_{t}^{\prime}\left(x_{t}\right) \prod_{s=t+1}^{T-1} \phi_{s}\left(x_{s}\right) g_{t-1}\left(\omega_{t-1} \mid z_{t-1}\right) d \omega_{T-1},
\end{aligned}
$$


where the second equality follows from integration by parts over $x_{t}$. When the feedback policy $f$ is even, we have

$$
\int_{\mathbf{R}^{T-1}} \sum_{s=t+1}^{T} c_{s}\left(\sigma_{s, 0}^{1}\left(z_{s-1}\right)\right) \phi_{t}^{\prime}\left(x_{t}\right) \prod_{s=t+1}^{T-1} \phi_{s}\left(x_{s}\right) g_{t-1}\left(\omega_{t-1} \mid z_{t-1}\right) d \omega_{T-1}=0 .
$$

To see this, for each subset $J$ of $\{2, \ldots, T-1\}$, let

$$
B(J)=\left\{\omega_{T-1} \in \mathbf{R}^{T-1}: x_{1}>0, x_{s}>0 \text { if } s \in J \text { and } x_{s}<0 \text { if } s \notin J\right\} .
$$

For example, $B(J)=\mathbf{R}_{+} \times\left(-\mathbf{R}_{+}^{T-2}\right)$ for $J=\phi$ and $B(J)=\mathbf{R}_{+}^{T-1}$ for $J=\{2, \ldots, T-1\}$. It can be seen that for each $J \subset\{2, \ldots, T-1\}$,

$$
\begin{aligned}
& \int_{B(J)} \sum_{s=t+1}^{T} c_{s}\left(\sigma_{s, 0}^{1}\left(z_{s-1}\right)\right) \phi_{t}^{\prime}\left(x_{t}\right) \prod_{s=t+1}^{T-1} \phi_{s}\left(x_{s}\right) g_{t-1}\left(\omega_{t-1} \mid z_{t-1}\right) d \omega_{T-1} \\
& +\int_{-B(J)} \sum_{s=t+1}^{T} c_{s}\left(\sigma_{s, 0}^{1}\left(z_{s-1}\right)\right) \phi_{t}^{\prime}\left(x_{t}\right) \prod_{s=t+1}^{T-1} \phi_{s}\left(x_{s}\right) g_{t-1}\left(\omega_{t-1} \mid z_{t-1}\right) d \omega_{T-1} \\
& =\int_{B(J)} \sum_{s=t+1}^{T} c_{s}\left(\sigma_{s, 0}^{1}\left(z_{s-1}\right)\right) \phi_{t}^{\prime}\left(x_{t}\right) \prod_{s=t+1}^{T-1} \phi_{s}\left(x_{s}\right) g_{t-1}\left(\omega_{t-1} \mid z_{t-1}\right) d \omega_{T-1} \\
& -\int_{-B(J)} \sum_{s=t+1}^{T} c_{s}\left(\sigma_{s, 0}^{1}\left(z_{s-1}\right)\right) \phi_{t}^{\prime}\left(-x_{t}\right) \prod_{s=t+1}^{T-1} \phi_{s}\left(-x_{s}\right) g_{t-1}^{\sigma}\left(-\omega_{t-1} \mid z_{t-1}\right) d \omega_{T-1} \\
& =\int_{B(J)} \sum_{s=t+1}^{T} c_{s}\left(\sigma_{s, 0}^{1}\left(z_{s-1}\right)\right) \phi_{t}^{\prime}\left(x_{t}\right) \prod_{s=t+1}^{T-1} \phi_{s}\left(x_{s}\right) g_{t-1}\left(\omega_{t-1} \mid z_{t-1}\right) d \omega_{T-1} \\
& -\int_{B(J)} \sum_{s=t+1}^{T} c_{s}\left(\sigma_{s, 0}^{1}\left(z_{s-1}\right)\right) \phi_{t}^{\prime}\left(x_{t}\right) \prod_{s=t+1}^{T-1} \phi_{s}\left(x_{s}\right) g_{t-1}\left(\omega_{t-1} \mid z_{t-1}\right) d \omega_{T-1} \\
& =0, \quad
\end{aligned}
$$

where the first equality uses $\phi_{s}\left(x_{s}\right)=\phi_{s}\left(-x_{s}\right)$ and $\phi_{t}^{\prime}\left(x_{t}\right)=-\phi_{t}^{\prime}\left(-x_{t}\right)$, and the second uses the change of variables and the fact that $z_{s-1}=\left(f_{1}\left(\omega_{1}\right), \ldots, f_{s-1}\left(\omega_{s-1}\right)\right)=$ $\left(f_{1}\left(-\omega_{1}\right), \ldots, f_{s-1}\left(-\omega_{s-1}\right)\right)$. (a27) follows if we note

$$
\int_{\mathbf{R}^{T-1}}=\sum_{J \subset\{2, \ldots, T-1\}}\left\{\int_{B(J)}+\int_{-B(J)}\right\} \text {. }
$$

From (a26) and (a27), we see that the stage $t$ effort on the symmetric equilibrium path should satisfy

$$
\sigma_{t, 0}^{1}\left(z_{t-1}\right)=\sigma_{t, 0}^{2}\left(z_{t-1}\right)=\left(c_{t}^{\prime}\right)^{-1}\left(E^{\sigma}\left[\phi_{T}\left(\tilde{\Delta}_{T-1}\right) \mid z_{t-1}\right]\right)=\alpha_{t}\left(z_{t-1}\right) .
$$


(a28) shows that (a24) and (a25) are equivalent to $\varphi_{t}^{1}\left(a_{t}^{1} \mid b_{t-1}^{1}, z_{t-1}\right)=0$ and $\varphi_{t}^{2}\left(a_{t}^{2} \mid\right.$ $\left.b_{t-1}^{2}, z_{t-1}\right)=0$, respectively.

Step 3. Finally, we show that the effort choice defined by $\varphi_{t}^{i}\left(a_{t}^{i} \mid b_{t-1}^{i}, z_{t-1}\right)=0$ maximizes each agent's payoff. For this, it suffices to verify that we have from Steps 1 and 2,

$$
\frac{\partial^{2} \pi_{t}^{i}}{\partial\left(a_{t}^{i}\right)^{2}}\left(a_{t}^{i} \mid b_{t-1}^{i}, z_{t-1}\right)=\frac{\partial \varphi_{t}^{i}}{\partial a_{t}^{i}}\left(a_{t}^{i} \mid b_{t-1}^{i}, z_{t-1}\right)<0
$$

for any $a_{t}^{i}$ and $t . / /$

Proof of TheOrem 12: Let $\sigma$ be the symmetric pure PBE under $f$ specified in (7). By the symmetry of $\sigma$, we have

$$
P^{\sigma}\left(\tilde{\Delta}_{t-1}=y_{t-1} \mid z_{t-1}\right)=P^{\sigma}\left(\tilde{\Delta}_{t-1}=-y_{t-1} \mid z_{t-1}\right)=\frac{1}{2} .
$$

It hence follows from (7) that

$$
\begin{aligned}
& c_{t}^{\prime}\left(\sigma_{t}^{i}\left(z_{t-1}\right)\right) \\
& =\int_{\mathbf{R}^{T-t}}\left\{\frac{1}{2} \phi_{T}\left(y_{t-1}+\sum_{s=t}^{T-1} x_{s}\right)+\frac{1}{2} \phi_{T}\left(-y_{t-1}+\sum_{s=t}^{T-1} x_{s}\right)\right\} \prod_{s=t}^{T-1} \phi_{s}\left(x_{s}\right) d x_{t} \cdots d x_{T-1} \\
& =\frac{1}{2}\left(\phi_{t} * \cdots * \phi_{T}\right)\left(-y_{t-1}\right)+\frac{1}{2}\left(\phi_{t} * \cdots * \phi_{T}\right)\left(y_{t-1}\right) \\
& =\left(\phi_{t} * \cdots * \phi_{T}\right)\left(y_{t-1}\right) .
\end{aligned}
$$

Therefore,

$$
\sigma_{t}^{i}\left(z_{t-1}\right)=\left(c_{t}^{\prime}\right)^{-1}\left(\left(\phi_{t} * \cdots * \phi_{T}\right)\left(y_{t-1}\right)\right)
$$

Now take any symmetric pure PBE $\sigma^{\prime}$ under any alternative feedback policy $f^{\prime}$. Denote $y_{t}^{\prime}=f_{t}^{\prime}\left(x_{1}, \ldots, x_{t}\right)$ and $z_{t}^{\prime}=\left(y_{1}^{\prime}, \ldots, y_{t}^{\prime}\right)$. Since $\left(c_{t}^{\prime}\right)^{-1}$ is concave, Jensen's inequality implies that

$$
\begin{aligned}
E^{\sigma^{\prime}}\left[\left(\sigma^{\prime}\right)_{t}^{i}\left(\tilde{z}_{t-1}^{\prime}\right)\right] & =E^{\sigma^{\prime}}\left[\left(c_{t}^{\prime}\right)^{-1}\left(E^{\sigma^{\prime}}\left[\phi_{T}\left(\tilde{\Delta}_{T-1}\right) \mid \tilde{z}_{t-1}^{\prime}\right]\right)\right] \\
& =E^{\sigma^{\prime}}\left[\left(c_{t}^{\prime}\right)^{-1}\left(E^{\sigma^{\prime}}\left[E^{\sigma^{\prime}}\left[\phi_{T}\left(\tilde{\Delta}_{T-1}\right) \mid \tilde{\omega}_{t-1}\right] \mid \tilde{z}_{t-1}^{\prime}\right]\right)\right] \\
& \leq E^{\sigma^{\prime}}\left[E^{\sigma^{\prime}}\left[\left(c_{t}^{\prime}\right)^{-1}\left(E^{\sigma^{\prime}}\left[\phi_{T}\left(\tilde{\Delta}_{T-1}\right) \mid \tilde{\omega}_{t-1}\right]\right) \mid \tilde{z}_{t-1}^{\prime}\right]\right] \\
& =E^{\sigma^{\prime}}\left[\left(c_{t}^{\prime}\right)^{-1}\left(E^{\sigma^{\prime}}\left[\phi_{T}\left(\tilde{\Delta}_{T-1}\right) \mid \tilde{\omega}_{t-1}\right]\right)\right] .
\end{aligned}
$$


On the other hand,

$$
\begin{aligned}
E^{\sigma^{\prime}}\left[\phi_{T}\left(\tilde{\Delta}_{T-1}\right) \mid \omega_{t-1}\right] & =\int_{\mathbf{R}^{T-t}} \phi_{T}\left(\Delta_{t-1}+\sum_{s=t}^{T-1} x_{s}\right) \prod_{s=t}^{T-1} \phi_{s}\left(x_{s}\right) d x_{t} \cdots d x_{T-1} \\
& =\left(\phi_{t} * \cdots * \phi_{T}\right)\left(\Delta_{t-1}\right) .
\end{aligned}
$$

Substituting this into the above, we obtain

$$
\begin{aligned}
E^{\sigma^{\prime}}\left[\left(\sigma^{\prime}\right)_{t}^{i}\left(\tilde{z}_{t-1}^{\prime}\right)\right] & \leq E^{\sigma^{\prime}}\left[\left(c_{t}^{\prime}\right)^{-1}\left(\left(\phi_{t} * \cdots * \phi_{T}\right)\left(\tilde{\Delta}_{t-1}\right)\right)\right] \\
& =\int_{\mathbf{R}^{t-1}}\left(c_{t}^{\prime}\right)^{-1}\left(\left(\phi_{t} * \cdots * \phi_{T}\right)\left(\left|\Delta_{t-1}\right|\right)\right) \prod_{s=1}^{t-1} \phi_{s}\left(x_{s}\right) d \omega_{t-1} \\
& =E^{\sigma}\left[\sigma_{t}^{i}\left(\tilde{z}_{t-1}\right)\right] .
\end{aligned}
$$

We hence obtain the desired conclusion. //

\section{References}

de-Frutos, M.-A. and R. Rosenthal (1998), "On some myths about sequenced commonvalue auctions," Games and Economic Behavior, 23, 201-21.

Fudenberg, D., R. Gilbert, J. Stiglitz and J. Tirole (1983), "Preemption, leapfrogging, and competition in patent races," European Economic Review, 22, 3-31.

Glazer, D. and R. Hassin (1988), "Optimal contests," Economic Inquiry, 26, 133-43.

Gradstein, M. and K. A. Konrad (1999), "Orchestrating rent seeking contests," Economic Journal, 109, 536-45.

Green, J. and N. Stokey (1983), "A comparison of tournaments and contracts," Journal of Political Economy, 91, 349-364.

Harris, C. and C. Vickers (1985), "Perfect equilibrium in a model of a race," Review of Economic Studies, 52, 193-209.

Kaplan, T. and S. Zamir (2000), "The strategic use of seller information in private-value auctions," mimeo., University of Exeter and the Hebrew University of Jerusalem.

Lazear, E. and S. Rosen (1981), "Rank order tournaments as optimal labor contracts," Journal of Political Economy, 89, 841-64.

Mares, V. and R. M. Harstad (2002), "Private information revelation in common-value auctions," forthcoming in the Journal of Economic Theory.

Milgrom, P. R. (1981), "Good news and bad news: Representation theorem with applications," Bell Journal of Economics, 21, 380-391. 
Milgrom, P. R. and R. Weber (1982), "A theory of auctions and competitive bidding," Econometrica, 50, 1059-1122.

Moldovanu, B. and A. Sela (2001), "The optimal allocation of prizes in contests," American Economic Review, 91, 542-58.

Nalebuff, B. J. and J. E. Stiglitz (1983), "Prizes and incentives: towards a general theory of compensation and competition," Bell Journal of Economics, 14, 21-43.

Perry, M. and P. J. Reny (1999), "On the failure of the linkage principle in multi-unit auctions," Econometrica, 67, 895-900.

Radner, R. (1985), "Repeated principal-agent games with discounting," Econometrica, 53, 1173-98.

Williams, R. (1998), Performance Management: Perspectives on Employee Performance, London: International Thomson Business Press. 